\title{
Statistical Analysis of a Weibull Extension with Bathtub-Shaped Failure Rate Function
}

\author{
Ronghua Wang, ${ }^{1}$ Naijun Sha, ${ }^{2}$ Beiqing Gu, ${ }^{3}$ and Xiaoling $\mathrm{Xu}^{3}$ \\ ${ }^{1}$ College of Mathematics and Science, Shanghai Normal University, Shanghai 200234, China \\ ${ }^{2}$ Department of Mathematical Sciences, University of Texas at El Paso, El Paso, TX 79968, USA \\ ${ }^{3}$ Business Information Management School, Shanghai University of International Business and Economics, Shanghai 201620, China
}

Correspondence should be addressed to Naijun Sha; nsha@utep.edu

Received 15 May 2014; Revised 11 September 2014; Accepted 6 October 2014; Published 27 October 2014

Academic Editor: Karthik Devarajan

Copyright (C) 2014 Ronghua Wang et al. This is an open access article distributed under the Creative Commons Attribution License, which permits unrestricted use, distribution, and reproduction in any medium, provided the original work is properly cited.

\begin{abstract}
We consider the parameter inference for a two-parameter life distribution with bathtub-shaped or increasing failure rate function. We present the point and interval estimations for the parameter of interest based on type-II censored samples. Through intensive Monte-Carlo simulations, we assess the performance of the proposed estimation methods by a comparison of precision. Example applications are demonstrated for the efficiency of the methods.
\end{abstract}

\section{Introduction}

In reliability analysis, the failure rate function plays an essential role to characterize life phenomena. The failure pattern of many products/systems (as electronic and mechanical products, etc.) can be represented by a bathtub curve. It comprises three stages: initial stage (or burn-in) with a decreasing failure rate, middle stage with an approximately constant failure rate, and final stage with an increasing failure rate. Many probability distributions have been proposed to fit real life data with bathtub-shaped failure rates (e.g., [15]). Chen [6] proposed a two-parameter lifetime distribution (written as $\operatorname{CZM}(\beta, \theta)$ throughout this paper) with bathtub failure rate whose distribution function is

$$
F(x)=1-\exp \left(\frac{1-e^{x^{\beta}}}{\theta}\right)
$$

where $\beta>0$ is the shape parameter and $\theta>0$ the scale parameter. Obviously, the transformed random variable $Y=$ $\left(e^{X^{\beta}}-1\right) / \theta$ follows the standard exponential distribution Exp (1). Also note that $F(x)=1-\exp \left(\left(1-e^{x^{\beta}}\right) / \theta\right)=1-\exp [1-$ $\left.\left(1+x^{\beta}+o\left(x^{\beta}\right)\right) / \theta\right] \approx 1-\exp \left(-x^{\beta} / \theta\right)$ for small $x$, and so the distribution $\operatorname{CZM}(\beta, \theta)$, to some degree, can be treated as an extension of Weibull distribution.
In reliability engineering, two common censoring mechanisms are widely applied in reliability testing for product life. For type-I censoring, the test is continued until a prespecified time is reached; whereas for type-II censoring, the test is carried out until a preset number $r$ of units failed. A significant amount of literature has emerged on various testing models with both data censoring schemes. Cha [7] considered a problem of determining optimal burn-in time in a bathtubshaped failure model under both cases of type-I and type-II. Xiong [8] made a statistical inference on a step-stress model with type-II censored exponential data. Balakrishnan et al. [9] presented the order restricted MLE for multiple step-stress models with exponential lifetime under type-I and type-II censored sampling situations. A comparison of accelerated life testing for Weibull and lognormal distributions with typeII censoring was made by meeker [10], just to name a few.

Particularly, for the $\operatorname{CZM}(\beta, \theta)$ distribution, there were some works done for parameter inference. Chen [6] presented the exact confidence intervals for the parameters based on type-II censored samples and gave an outline of the maximum likelihood estimation (MLE) procedure. Wu et al. [11] discussed the optimal pivotal quantities to provide a shortest exact confidence interval of the parameters and presented an alternate approximated one. Tang et al. [12] extended $\operatorname{CZM}(\beta, \theta)$ to a three-parameter distribution, for 
which they studied the properties of the order statistics, and introduced the MLE estimation and likelihood ratio test. However, in general, this distribution has not been much explored for its characteristics and parameter inference.

In this paper, we will explore some features of $\operatorname{CZM}(\beta, \theta)$ and primarily focus on the parameter inference. In Section 2, we first study the numerical and graphic characteristics of this distribution and correct some mistakes which appeared in Chen [6] and Tang et al. [12]. We present several methods of point and interval estimation for the parameters in Section 3. In Section 4 we assess the performance of the methods by comparing the estimation precision on simulation studies, followed by data examples in Section 5. Lastly we conclude the paper with a brief discussion in Section 6.

\section{Characteristics of Distribution $\operatorname{CZM}(\beta, \theta)$}

The density function for the distribution in (1) is

$$
f(x)=\frac{\beta}{\theta} x^{\beta-1} e^{x^{\beta}} \exp \left(\frac{1-e^{x^{\beta}}}{\theta}\right), \quad x>0,
$$

which has some interesting characteristics illustrated in Lemma 1.

Lemma 1. The density function $f(x)$ has the following characteristics. (1) When $\beta=1, f(x)$ is decreasing for $0<\theta \leq 1$, and unimodal for $\theta>1$. (2) When $\beta>1, f(x)$ is unimodal. (3) When $0<\beta<1$, (i) $f(x)$ is decreasing for $0<\theta \leq 1$; (ii) if $\theta>1$, then $f(x)$ is either decreasing, or first decreasing, then increasing and finally decreasing again.

We provide a detail proof in the Appendix. Note that Lemma 1 corrects the wrong conclusion of Tang et al. [12], who claimed that the density $f(x)$ was decreasing with $\beta=$ $1, \theta>1$. Figure 1 shows the density curves under various settings of parameter values. It is also worth noting that when $\beta=1$, the distribution becomes an extreme value distribution, and when $\theta=1$, it reduces to an exponential power life distribution introduced by Dhillon [13], which can have increasing, decreasing, and bathtub-shaped failure rates. Hence, it seems that the distribution is flexible enough to model various situations of product life.

The failure rate function is $\lambda(x)=(\beta / \theta) x^{\beta-1} e^{x^{\beta}}$, and $\lambda^{\prime}(x)=(\beta / \theta) x^{\beta-2} e^{x^{\beta}}\left[(\beta-1)+\beta x^{\beta}\right], x>0$, thus $\lambda(x)$ has a bathtub-shaped with achieving a minimum value at $x=((1-\beta) / \beta)^{1 / \beta}$ when $0<\beta<1$, and is increasing when $\beta \geq 1$. Figure 2 illustrates $\lambda(x)$ with various values of $\beta$ and $\theta$. Since the bottom of bathtub is the steady stage of failure rate function, it corresponds to the regular working stage of products and is very important for the practical application. To describe the length of time for working stage, we first give the definition of bathtub bottom width in the following.

Definition 2. Suppose that a random variable $X$ follows a bathtub-shaped failure rate function $\lambda(x)$ with achieving the minimum at $x_{0}$. For a given (bathtub height) $\varepsilon>0$ and two roots $x_{1}, x_{2}$ of the equation $\lambda(x)-\lambda\left(x_{0}\right)=\varepsilon$ with $x_{1}<x_{0}<$ $x_{2}$, define the difference $l(\varepsilon)=x_{2}-x_{1}$ as the bathtub bottom width of the given precision $\varepsilon$.

For $\operatorname{CZM}(\beta, \theta)$ distribution, we have the following property of the bottom width for the failure rate function.

Theorem 3. For $C Z M(\beta, \theta)$ with $0<\beta<1$ and the bathtubshaped failure rate function $\lambda(x)$, then given $\varepsilon>0$, the bathtub bottom width $l(\varepsilon)$ is increasing for $\theta$.

Proof. Since $\lambda(x)$ has a bathtub-shaped for $0<\beta<1$, given $\varepsilon>0$, there are two roots $x_{1}<x_{2}$ of the equation $\lambda(x)-$ $\lambda\left(x_{0}\right)=\varepsilon$ with $x_{0}=((1-\beta) / \beta)^{1 / \beta}$. Taking derivative of the equation $\lambda(x)-\lambda\left(x_{0}\right)=\varepsilon$ with respect to $\theta$, we have $\partial x / \partial \theta=$ $\varepsilon / \beta x^{\beta-2} e^{x^{\beta}}\left[\beta-1+\beta x^{\beta}\right]$. Let $g(x)=1 / x^{\beta-2} e^{x^{\beta}}\left[\beta-1+\beta x^{\beta}\right]$, obviously $g\left(x_{1}\right)<0<g\left(x_{2}\right)$ since $x_{1}<x_{0}<x_{2}$. Thus, we obtain $\partial l(\varepsilon) / \partial \theta=\partial\left(x_{2}-x_{1}\right) / \partial \theta=(\varepsilon / \beta)\left[g\left(x_{2}\right)-g\left(x_{1}\right)\right]>0$. The theorem follows.

Given $\varepsilon=0.1,0.01,0.001,0.0001$, and under various values of distribution parameters, the results of the bottom width are listed in Table 1, in which one can see that the width $l(\varepsilon)$ is increasing for $\theta$, on the contrary, $l(\varepsilon)$ is decreasing for $\beta$.

For the purpose of estimation, we explore other characteristics of the distribution including finite moments. Finite moments guarantee the rationality for the moment-based estimation methods such as method of moments. We display our findings for the properties of $\operatorname{CZM}(\beta, \theta)$ shown in Theorem 4 whose proof is provided in the Appendix.

Theorem 4. Suppose $X \sim \operatorname{CZM}(\beta, \theta)$. Then, (1) the moments exist; (2) the average failure rate $G(x)=-(\log (1-$ $F(x))) / x, x>0$ is increasing for $\beta \geq 1$ and has a bathtubshaped (concave-up) for $0<\beta<1$; (3) the average remaining lifetime function $m(x)=\left(\int_{x}^{+\infty}[1-F(t)] d t\right) /(1-F(x)), x>0$ is decreasing for $\beta \geq 1$, and unimodal for $0<\beta<1$.

Not surprisingly, there is a similar characteristic for the failure rate $\lambda(x)$ and average failure rate $G(x)$. Figures 3 and 4 illustrate the shapes of $G(x)$ and $m(x)$ with various values of $\beta$.

\section{Estimation Methods}

In this section, we discuss the estimation of parameters under type-II censored observational data. Suppose that $n$ products whose lifetime follow $\operatorname{CZM}(\beta, \theta)$ are placed on test and the test continues until predetermined $r$ products fail with the ordered failure times $x_{(1)} \leq x_{(2)} \leq \cdots \leq x_{(r)}$. We present the methods of point estimation for $\beta, \theta$ and the interval estimation only for the shape parameter $\beta$.

3.1. Maximum Likelihood Estimation. Under the above typeII censoring, the likelihood function is

$L(\beta, \theta)$

$$
=\frac{n !}{(n-r) !}\left(\prod_{i=1}^{r} f\left(x_{(i)}\right)\right)\left[1-F\left(x_{(r)}\right)\right]^{n-r}
$$




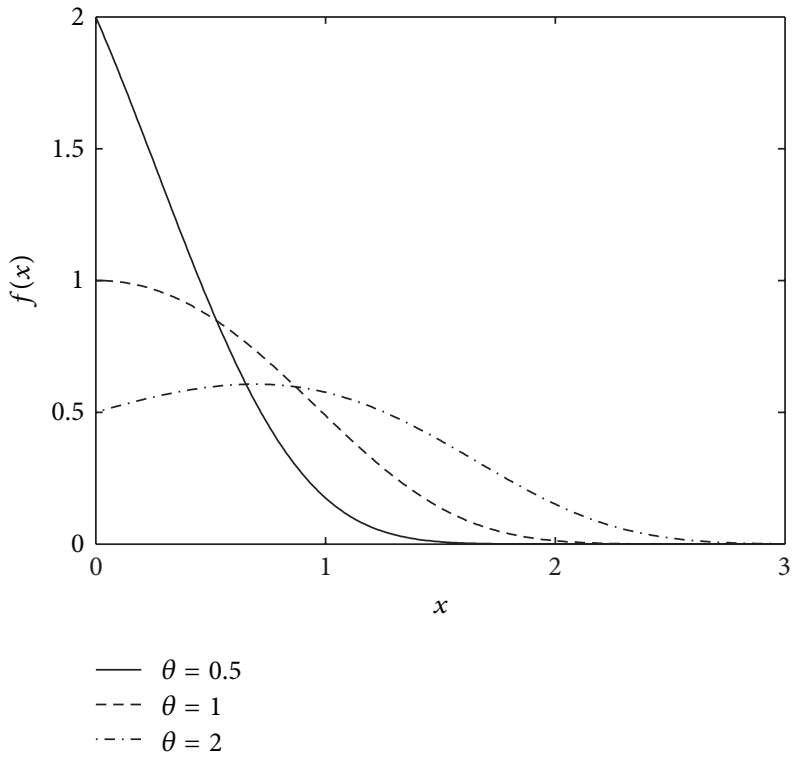

(a) $\beta=1$

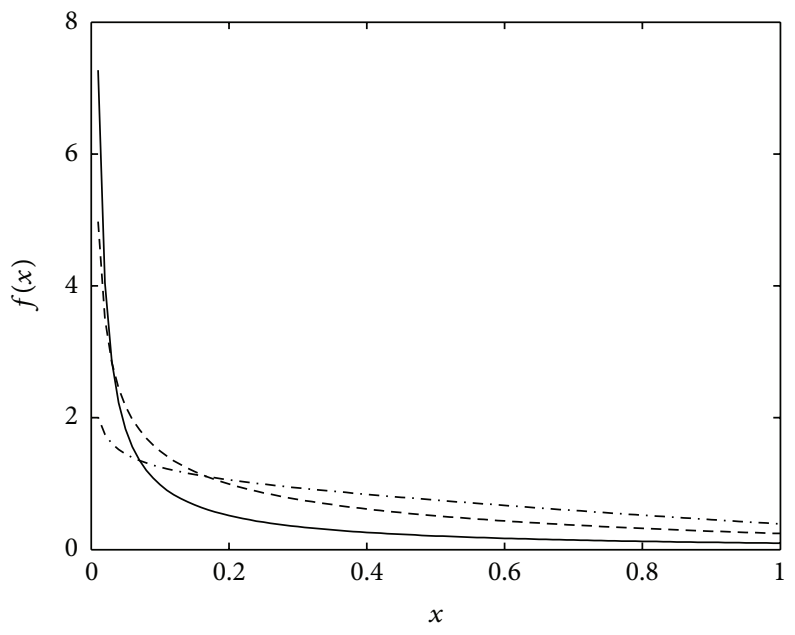

$\begin{aligned}-\beta & =0.2 \\ --\beta & =0.5 \\ -\beta & =0.8\end{aligned}$

(c) $\theta=1,0<\beta<1$

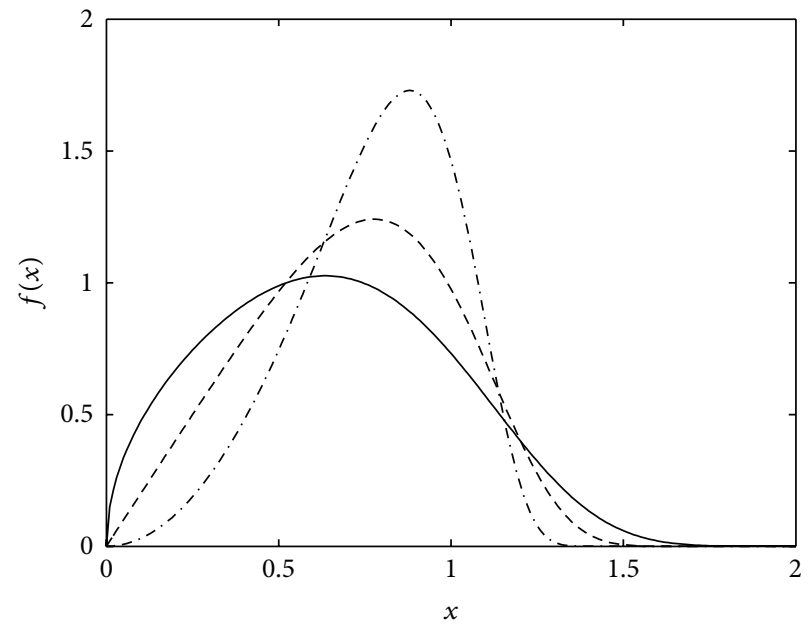

$-\beta=1.5$

- - $\beta=2$

.. $\beta=3$

(b) $\theta=1, \beta>1$

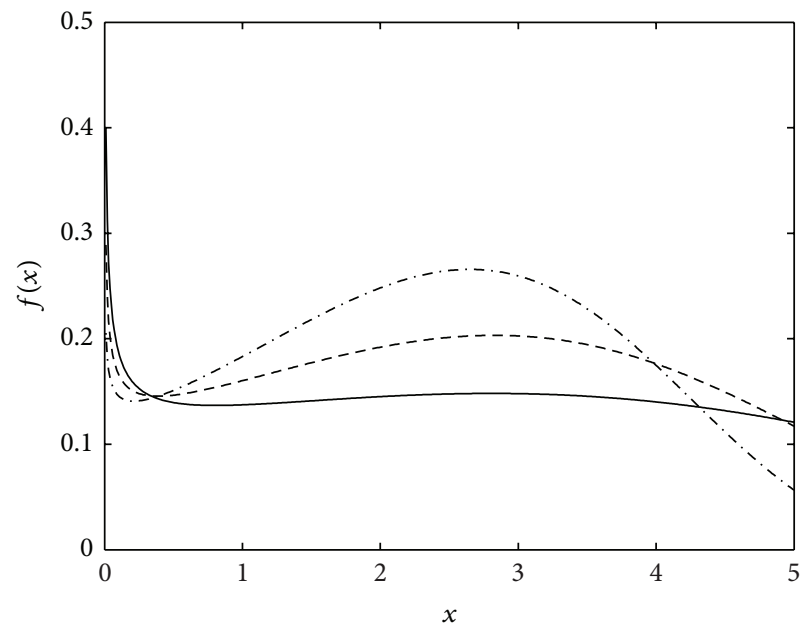

$-\beta=0.6$
$--\beta=0.7$
$\cdots-\beta=0.8$

(d) $\theta=10,0<\beta<1$

FIGURE 1: Density function $f(x)$.

$$
\begin{aligned}
= & \frac{n !}{(n-r) !} \frac{\beta^{r}}{\theta^{r}}\left(\prod_{i=1}^{r} x_{(i)}\right)^{\beta-1} \\
& \times \exp \left[\sum_{i=1}^{r} x_{(i)}^{\beta}+\frac{1}{\theta} \sum_{i=1}^{r}\left(1-e^{x_{(i)}^{\beta}}\right)+\frac{(n-r)}{\theta}\left(1-e^{x_{(r)}^{\beta}}\right)\right] .
\end{aligned}
$$

The partial derivatives of $\log$-likelihood equations $(\partial \log L(\beta$, $\theta)) / \partial \beta=0,(\partial \log L(\beta, \theta)) / \partial \theta=0$ give us

$$
\theta=\frac{1}{r}\left[\sum_{i=1}^{r}\left(e^{x_{(i)}^{\beta}}-1\right)+(n-r)\left(e^{x_{(r)}^{\beta}}-1\right)\right],
$$

$$
\begin{gathered}
\frac{\sum_{i=1}^{r} x_{(i)}^{\beta} e^{x_{(i)}^{\beta}} \log x_{(i)}+(n-r) x_{(r)}^{\beta} e^{x_{(r)}^{\beta}} \log x_{(r)}}{\sum_{i=1}^{r}\left(e^{x_{(i)}^{\beta}}-1\right)+(n-r)\left(e^{x_{(r)}^{\beta}}-1\right)} \\
-\frac{1}{r} \sum_{i=1}^{r} x_{(i)}^{\beta} \log x_{(i)}-\frac{1}{r} \sum_{i=1}^{r} \log x_{(i)}-\frac{1}{\beta}=0 .
\end{gathered}
$$

The maximum likelihood estimate (MLE) for $\theta$ is $\widehat{\theta}_{1}=$ $(1 / r)\left[\sum_{i=1}^{r}\left(e^{x_{(i)}^{\hat{\beta}_{1}}}-1\right)+(n-r)\left(e^{x_{(r)}^{\hat{\beta}_{1}}-1}\right)\right]$, where no analytic form of MLE $\widehat{\beta}_{1}$ can be obtained, and a numeric method has to be applied. The uniqueness of MLE can be shown by using the 

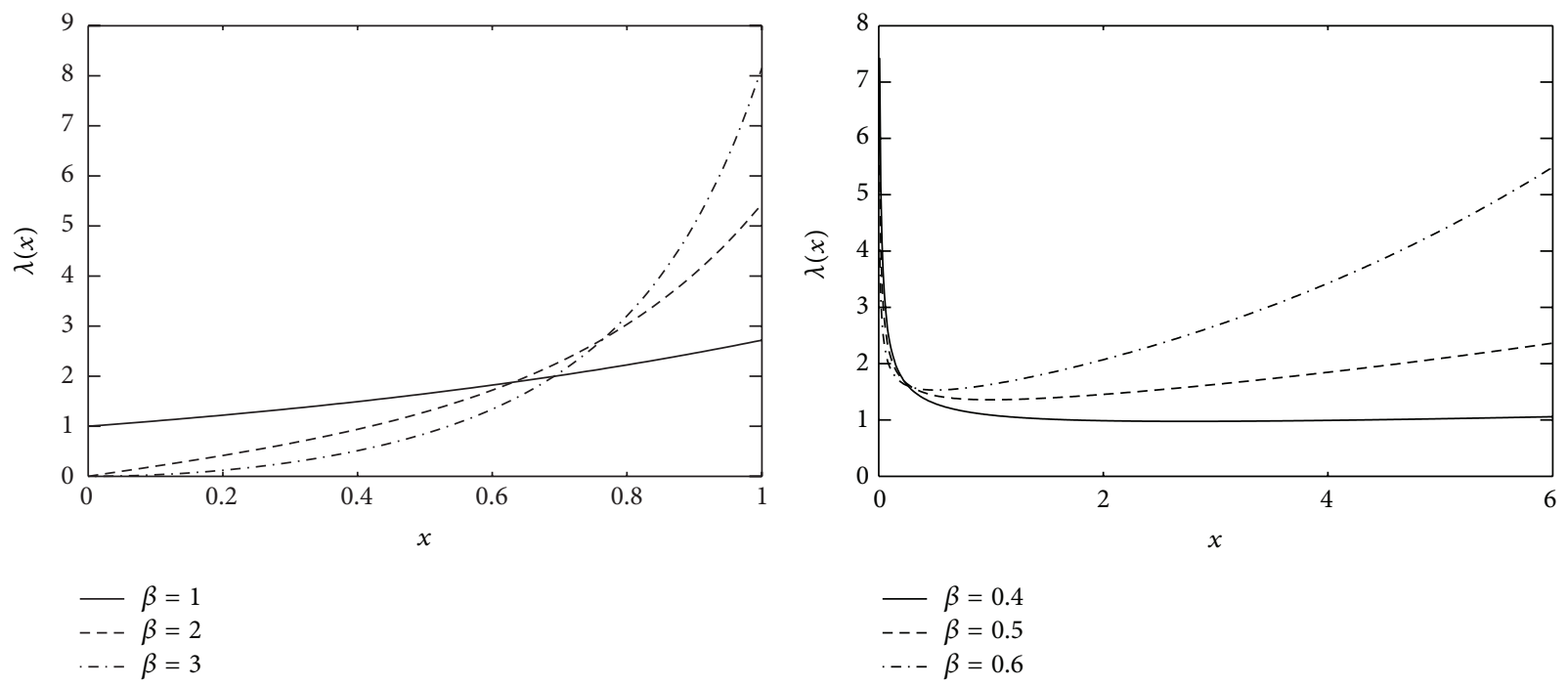

(a) $\theta=1, \beta \geq 1$

(b) $\theta=1,0<\beta<1$

FIgURE 2: Failure rate function $\lambda(x)$.

TABLE 1: The bathtub bottom width.

\begin{tabular}{|c|c|c|c|c|c|c|c|c|}
\hline \multicolumn{5}{|c|}{$\beta=0.5$} & \multicolumn{4}{|c|}{$\theta=0.5$} \\
\hline$\varepsilon$ & $\theta$ & $x_{1}$ & $x_{2}$ & $l(\varepsilon)=x_{2}-x_{1}$ & $\beta$ & $x_{1}$ & $x_{2}$ & $l(\varepsilon)=x_{2}-x_{1}$ \\
\hline \multirow{3}{*}{0.1} & 0.5 & 0.5696 & 1.6729 & 1.1033 & 0.3 & 5.7180 & 44.6868 & 38.9688 \\
\hline & 1 & 0.4475 & 2.0322 & 1.5847 & 0.5 & 0.5696 & 1.6729 & 1.1033 \\
\hline & 2 & 0.3172 & 2.6219 & 2.3047 & 0.8 & 0.0867 & 0.3216 & 0.2349 \\
\hline \multirow{3}{*}{0.01} & 0.5 & 0.8404 & 1.1841 & 0.3437 & 0.3 & 12.0165 & 23.3669 & 11.3504 \\
\hline & 1 & 0.7810 & 1.2679 & 0.4869 & 0.5 & 0.8404 & 1.1841 & 0.3437 \\
\hline & 2 & 0.7034 & 1.3943 & 0.6909 & 0.8 & 0.1428 & 0.2163 & 0.0734 \\
\hline \multirow{3}{*}{0.001} & 0.5 & 0.9470 & 1.0555 & 0.1085 & 0.3 & 15.1556 & 18.7121 & 3.5565 \\
\hline & 1 & 0.9257 & 1.0792 & 0.1535 & 0.5 & 0.9470 & 1.0555 & 0.1085 \\
\hline & 2 & 0.8963 & 1.1135 & 0.2171 & 0.8 & 0.1655 & 0.1887 & 0.0232 \\
\hline \multirow{3}{*}{0.0001} & 0.5 & 0.9830 & 1.0173 & 0.0343 & 0.3 & 16.2962 & 17.4198 & 1.1236 \\
\hline & 1 & 0.9760 & 1.0245 & 0.0485 & 0.5 & 0.9830 & 1.0173 & 0.0343 \\
\hline & 2 & 0.9662 & 1.0348 & 0.0686 & 0.8 & 0.1731 & 0.1805 & 0.0073 \\
\hline
\end{tabular}

following Lemma 5 presented in Chen [6] (in which a wrong proof was given). We provide a correct proof of the lemma as well as the uniqueness of MLE in the appendix.

Lemma 5. Let $\xi(\beta)=\left(e^{b^{\beta}}-1\right) /\left(e^{a^{\beta}}-1\right)$ with the constants $a>0, b>0$, then

(1)

$$
\lim _{\beta \rightarrow+\infty} \xi(\beta)= \begin{cases}1, & a=b \\ 0, & a>b \\ +\infty, & a<b\end{cases}
$$

(2) For $b>a>0, \xi(\beta)$ is strictly increasing in $\beta>0$.
3.2. Method of Moment Estimation. It is known that $Y_{(i)}=$ $\left(e^{X_{(i)}^{\beta}}-1\right) / \theta, i=1,2, \ldots, r$ are the first $r$ ordered statistics of $n$ random samples from $\operatorname{Exp}(1)$, and the mean and variance of $Y_{(i)}$ are $a_{i, n}=\sum_{k=1}^{i}(1 /(n-k+1))$ and $d_{i, n}=\sum_{k=1}^{i}\left(1 /(n-k+1)^{2}\right)$, respectively [14]. We set up the following equations by the first two "ordered" moments to obtain method of moment (MOM) estimates as follows:

$$
\begin{aligned}
& \sum_{i=1}^{r}\left(\frac{e^{x_{(i)}^{\beta}}-1}{\theta}\right)=\sum_{i=1}^{r} a_{i, n}, \\
& \sum_{i=1}^{r}\left(\frac{e^{x_{(i)}^{\beta}}-1}{\theta}\right)^{2}=\sum_{i=1}^{r} b_{i, n},
\end{aligned}
$$




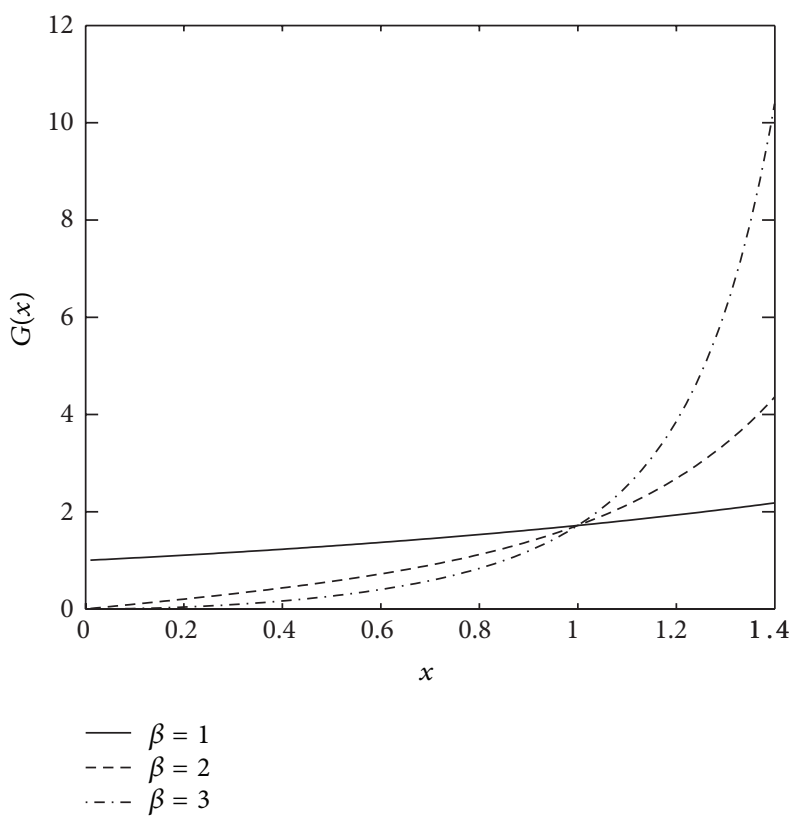

(a) $\theta=1, \beta \geq 1$

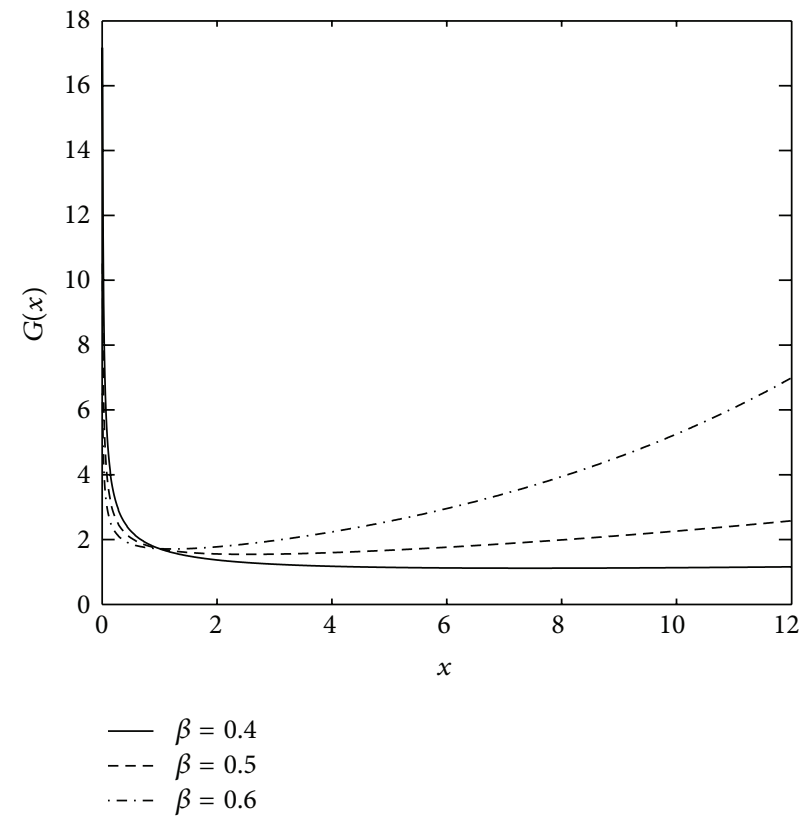

(b) $\theta=1,0<\beta<1$

FIGURE 3: Average failure function $G(x)$.
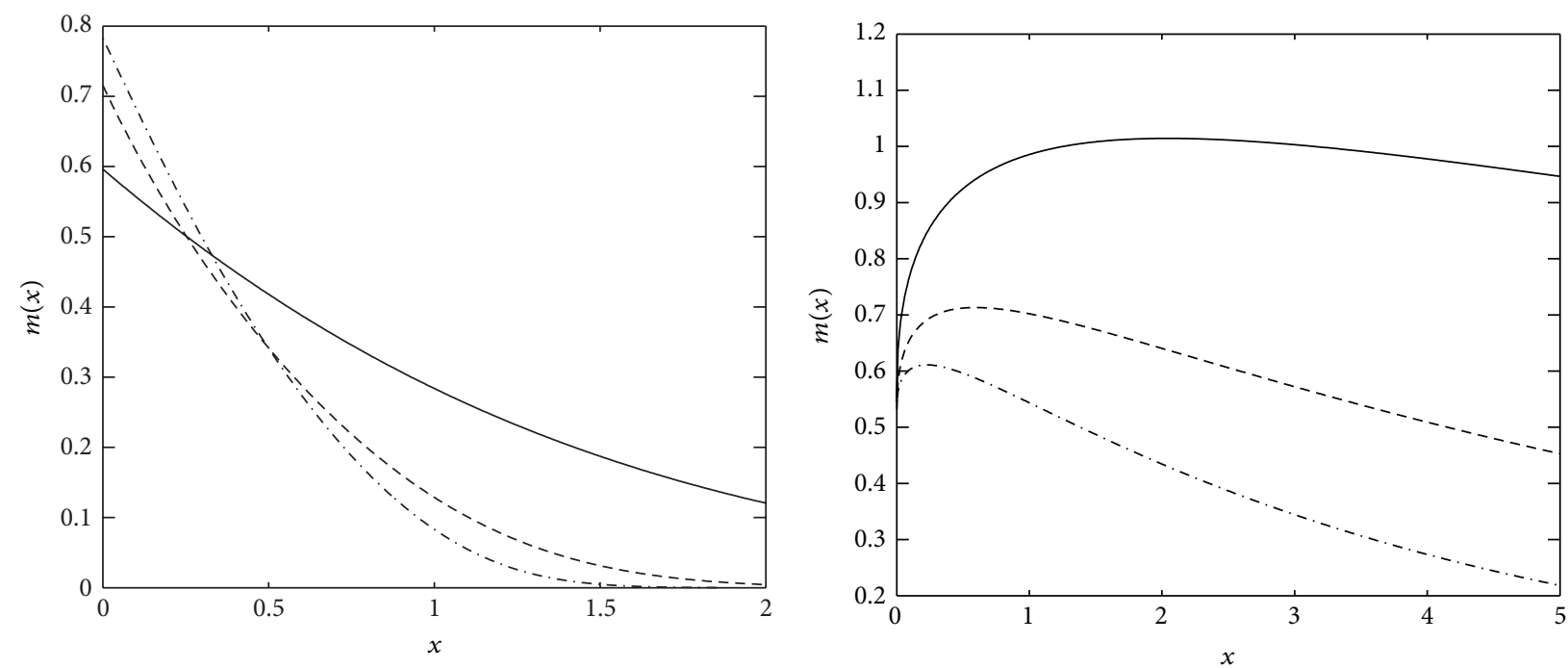

$$
\begin{aligned}
- & \beta=1 \\
-- & \beta=2 \\
-- & \beta=3
\end{aligned}
$$$$
\begin{aligned}
-\beta & =0.4 \\
--\beta & =0.5 \\
--\beta & =0.6
\end{aligned}
$$

(a) $\theta=1, \beta \geq 1$

(b) $\theta=1,0<\beta<1$

FIGURE 4: Average remaining lifetime function $m(x)$. 
where $b_{i, n}=a_{i, n}^{2}+d_{i, n}$. Therefore, the MOM estimate is $\widehat{\theta}_{2}=\left(\sum_{i=1}^{r}\left(e^{x_{(i)}^{\hat{\beta}_{2}}}-1\right)\right) / \sum_{i=1}^{r} a_{i, n}$ with $\widehat{\beta}_{2}$ being the root of $\left(\sum_{i=1}^{r}\left(e^{x_{(i)}^{\beta}}-1\right)^{2}\right) /\left[\sum_{i=1}^{r}\left(e^{x_{(i)}^{\beta}}-1\right)\right]^{2}=\left(\sum_{i=1}^{r} b_{i, n}\right) /\left(\sum_{i=1}^{r} a_{i, n}\right)^{2}$. The uniqueness of $\widehat{\beta}_{2}$ is shown in the appendix. In addition

$$
a(n, r)=\frac{\sum_{i=1}^{r}\left(e^{X_{(i)}^{\beta}}-1\right)^{2}}{\left[\sum_{i=1}^{r}\left(e^{X_{(i)}^{\beta}}-1\right)\right]^{2}}=\frac{\sum_{i=1}^{r} Y_{(i)}^{2}}{\left[\sum_{i=1}^{r} Y_{(i)}\right]^{2}}
$$

is a pivotal quantity (called MOM quantity) whose distribution is unknown. Under the different values of sample size $n$ and number of failures $r$, we carry out 10000 Monte-Carlo simulations to compute some quantiles of the pivotal quantity $a(n, r)$ and the mean $\bar{a}(n, r)$, listed in Table 2 . Then, we suggest a modified method of moment (MMOM) estimate $\widehat{\beta}_{3}$, the root of equation $\left(\sum_{i=1}^{r}\left(e^{x_{(i)}^{\beta}}-1\right)^{2}\right) /\left[\sum_{i=1}^{r}\left(e^{x_{(i)}^{\beta}}-1\right)\right]^{2}=\bar{a}(n, r)$, and accordingly $\widehat{\theta}_{3}=\left(\sum_{i=1}^{r}\left(e^{x_{(i)}^{\hat{\beta}_{3}}}-1\right)\right) / \sum_{i=1}^{r} a_{i, n}$.

Furthermore, using this pivotal quantity, the $100 \times(1-$ $\alpha) \%$ confidence interval for $\beta$ is constructed from the roots of the equation $a(n, r)=c$ with $c$ the lower and upper $(\alpha / 2)$ th quantiles under various settings of $(n, r)$ in Table 2 .

3.3. Inverse Method of Moment Estimation. It is well known that $(n-i+1)\left(Y_{(i)}-Y_{(i-1)}\right), i=1,2, \ldots, r$ with $Y_{(0)}=0$ is a random sample from $\operatorname{Exp}(1)$ [15], we define

$$
\begin{array}{r}
S_{k}=\sum_{i=1}^{k}(n-i+1)\left(Y_{(i)}-Y_{(i-1)}\right) \\
=\frac{1}{\theta}\left[\sum_{i=1}^{k}\left(e^{X_{(i)}^{\beta}}-1\right)+(n-k)\left(e^{X_{(k)}^{\beta}}-1\right)\right], \\
k=1,2, \ldots, r .
\end{array}
$$

By using the fact that $2 S_{r} \sim \chi_{2 r}^{2}, 2 \sum_{k=1}^{r-1} \log \left(S_{r} / S_{k}\right) \sim$ $\chi_{2 r-2}^{2}$ [16], we set up the equations by their first moments $2 \sum_{k=1}^{r-1} \log \left(S_{r} / S_{k}\right)=2 r-2$ to get the root $\widehat{\beta}_{4}$ and $2 S_{r}=2 r$ to get $\widehat{\theta}_{4}=(1 / r)\left[\sum_{i=1}^{r}\left(e^{x_{(i)}^{\hat{\beta}_{4}}}-1\right)+(n-r)\left(e^{x_{(r)}^{\hat{\beta}_{4}}}-1\right)\right]$, termed as inverse method of moment (IMOM) estimates. In the case of small sample size $n$, we set up the equations by the distribution modes $2 S_{r}=2(r-1), 2 \sum_{k=1}^{r-1} \log \left(S_{r} / S_{k}\right)=2(r-2)$ to get the modified inverse moment (MIM) estimates $\widehat{\beta}_{5}$ and $\widehat{\theta}_{5}=$ $(1 /(r-1))\left[\sum_{i=1}^{r}\left(e^{x_{(i)}^{\hat{\beta}_{5}}}-1\right)+(n-r)\left(e^{x_{(r)}^{\hat{\beta}_{5}}}-1\right)\right]$.

The pivotal quantity $2 \sum_{k=1}^{r-1} \log \left(S_{r} / S_{k}\right)$ (called MIM quantity) may result in a $(1-\alpha) \times 100 \%$ confidence interval for $\beta$ with the lower and upper limits from the equations

$$
2 \sum_{k=1}^{r-1} \log \frac{S_{r}}{S_{k}}=c
$$

with $c=\chi_{\alpha / 2,2 r-2}^{2}, \chi_{1-\alpha / 2,2 r-2}^{2}$.
In what follows, we prove that the estimators $\widehat{\beta}_{4}$ and $\widehat{\beta}_{5}$ obtained above are unique. Note that

$$
\begin{aligned}
& \frac{S_{r}}{S_{k}}=1+\left(\left(\sum_{i=k+1}^{r} \frac{e^{x_{(i)}^{\beta}}-1}{e^{x_{(k)}^{\beta}}-1}+(n-r)\right.\right. \\
& \left.\times \frac{e^{x_{(r)}^{\beta}}-1}{e^{x_{(k)}^{\beta}}-1}-(n-k)\right) \\
& \left.\times\left(\sum_{i=1}^{k-1} \frac{e^{x_{(i)}^{\beta}}-1}{e^{x_{(k)}^{\beta}}-1}+(n-k+1)\right)^{-1}\right), \\
& k=1,2, \ldots, r-1 \text {. }
\end{aligned}
$$

By Lemma 5, $\left(e^{x_{(i)}^{\beta}}-1\right) /\left(e^{x^{\beta}}(k)-1\right)$ is strictly increasing of $\beta$ for $i>k$ and decreasing for $i<k$. Then, $S_{r} / S_{k}$ is strictly increasing, so is $\sum_{k=1}^{r-1} \log \left(S_{r} / S_{k}\right)$. In addition, $\lim _{\beta \rightarrow 0}\left(\left(e^{x_{(i)}^{\beta}}-\right.\right.$ $\left.1) /\left(e^{x_{(k)}^{\beta}}-1\right)\right)=1$, then $\lim _{\beta \rightarrow 0}\left(S_{r} / S_{k}\right)=1$, and thus $\lim _{\beta \rightarrow 0} \sum_{k=1}^{r-1} \log \left(S_{r} / S_{k}\right)=0$. Also since

$$
\lim _{\beta \rightarrow+\infty} \frac{e^{x_{(i)}^{\beta}}-1}{e^{x_{(k)}^{\beta}}-1}= \begin{cases}0, & i<k \\ +\infty, & i>k\end{cases}
$$

we have $\lim _{\beta \rightarrow+\infty}\left(S_{r} / S_{k}\right)=+\infty$, and thus $\lim _{\beta \rightarrow+\infty} \sum_{k=1}^{r-1} \log \left(S_{r} / S_{k}\right)=+\infty$. Hence, for any $a>0$ (specifically for our case here, $a=r-1>0$ or $a=r-2>0$ ), the equation $\sum_{k=1}^{r-1} \log \left(S_{r} / S_{k}\right)=a$ has a unique root in $\beta>0$.

An exact confidence interval was provided in $\mathrm{Wu}$ et al. [11], who employed the fact that the pivotal quantities (called Wu's exact quantity) $\left(2\left(S_{r}-S_{k}\right) / 2(r-k)\right) /\left(2 S_{k} / 2 k\right)=(k /(r-$ $k))\left(\left(S_{r}-S_{k}\right) / S_{k}\right) \sim F_{2(r-k), 2 k}, k=1,2, \ldots, r-1$ to form the $k$ th $100 \times(1-\alpha) \%$ confidence interval for $\beta$ with the low and upper limits being the solutions from the following equation

$$
\frac{k}{r-k} \frac{S_{r}-S_{k}}{S_{k}}=c,
$$

where $c=F_{\alpha / 2,2(r-k), 2 k}, F_{1-\alpha / 2,2(r-k), 2 k}$. The optimal interval is the one with the shortest width among the $r-1$ intervals. Notice that the interval with $k=1$ reduces to the one constructed in Chen [6]. Wu et al. [11] also provided an approximated interval estimation by using the idea of Mann et al. [17] through the following pivotal quantities (called Wu's approx. quantity):

$$
\frac{k\left(Y_{(r)}-Y_{(k)}\right)}{m S_{k}} \stackrel{\text { approx. }}{\sim} F_{2 m^{2} / v, 2 k}, \quad k=1,2, \ldots, r-1,
$$

with $m=\sum_{i=k+1}^{r}(1 /(n-i+1)), v=\sum_{i=k+1}^{r}(1 /(n-i+$ $\left.1)^{2}\right)$. Again, the optimal is the interval with the shortest width among the $r-1$ intervals.

To determine what value of $k$, in general, results in the optimal for Wu's methods, we conduct a simulation study under various settings of $(n, r)$. We only show the results for Wu's exact interval since similar findings were obtained for 
TABLE 2: Some quantiles of pivotal quantity $a(n, r)$ and mean $\bar{a}(n, r)$ in 10000 simulations.

\begin{tabular}{|c|c|c|c|c|c|c|c|c|c|c|}
\hline$n$ & $r$ & 0.995 & 0.975 & 0.95 & 0.90 & 0.10 & 0.05 & 0.025 & 0.005 & $\bar{a}(n, r)$ \\
\hline \multirow{3}{*}{10} & 8 & 0.1363 & 0.1437 & 0.1484 & 0.1545 & 0.2375 & 0.2592 & 0.2820 & 0.3361 & 0.1921 \\
\hline & 9 & 0.1251 & 0.1324 & 0.1370 & 0.1433 & 0.2255 & 0.2467 & 0.2692 & 0.3232 & 0.1803 \\
\hline & 10 & 0.1178 & 0.1259 & 0.1311 & 0.1378 & 0.2367 & 0.2649 & 0.2927 & 0.3638 & 0.1819 \\
\hline \multirow{5}{*}{15} & 11 & 0.1022 & 0.1072 & 0.1104 & 0.1144 & 0.1628 & 0.1740 & 0.1851 & 0.2117 & 0.1365 \\
\hline & 12 & 0.0956 & 0.1005 & 0.1035 & 0.1073 & 0.1534 & 0.1640 & 0.1747 & 0.1998 & 0.1285 \\
\hline & 13 & 0.0902 & 0.0951 & 0.0980 & 0.1018 & 0.1473 & 0.1582 & 0.1692 & 0.1951 & 0.1227 \\
\hline & 14 & 0.0862 & 0.0913 & 0.0944 & 0.0983 & 0.1461 & 0.1577 & 0.1695 & 0.1984 & 0.1202 \\
\hline & 15 & 0.0841 & 0.0898 & 0.0933 & 0.0978 & 0.1585 & 0.1750 & 0.1922 & 0.2333 & 0.1250 \\
\hline \multirow{5}{*}{20} & 16 & 0.0740 & 0.0775 & 0.0796 & 0.0824 & 0.1123 & 0.1189 & 0.1252 & 0.1403 & 0.0963 \\
\hline & 17 & 0.0708 & 0.0744 & 0.0765 & 0.0792 & 0.1088 & 0.1154 & 0.1217 & 0.1363 & 0.0930 \\
\hline & 18 & 0.0684 & 0.0720 & 0.0742 & 0.0768 & 0.1071 & 0.1139 & 0.1204 & 0.1363 & 0.0908 \\
\hline & 19 & 0.0665 & 0.0704 & 0.0726 & 0.0754 & 0.1081 & 0.1156 & 0.1230 & 0.1405 & 0.0905 \\
\hline & 20 & 0.0661 & 0.0702 & 0.0728 & 0.0760 & 0.1185 & 0.1297 & 0.1410 & 0.1690 & 0.0952 \\
\hline \multirow{6}{*}{25} & 20 & 0.0605 & 0.0633 & 0.0649 & 0.0670 & 0.0885 & 0.0928 & 0.0970 & 0.1067 & 0.0770 \\
\hline & 21 & 0.0586 & 0.0613 & 0.0629 & 0.0649 & 0.0861 & 0.0905 & 0.0948 & 0.1040 & 0.0748 \\
\hline & 22 & 0.0568 & 0.0596 & 0.0612 & 0.0632 & 0.0846 & 0.0890 & 0.0933 & 0.1031 & 0.0732 \\
\hline & 23 & 0.0555 & 0.0584 & 0.0600 & 0.0620 & 0.0842 & 0.0890 & 0.0937 & 0.1042 & 0.0723 \\
\hline & 24 & 0.0544 & 0.0575 & 0.0593 & 0.0614 & 0.0857 & 0.0911 & 0.0964 & 0.1095 & 0.0727 \\
\hline & 25 & 0.0546 & 0.0580 & 0.0600 & 0.0625 & 0.0942 & 0.1022 & 0.1102 & 0.1312 & 0.0769 \\
\hline \multirow{7}{*}{30} & 24 & 0.0513 & 0.0536 & 0.0549 & 0.0565 & 0.0729 & 0.0762 & 0.0792 & 0.0860 & 0.0642 \\
\hline & 25 & 0.0499 & 0.0522 & 0.0534 & 0.0550 & 0.0711 & 0.0744 & 0.0774 & 0.0841 & 0.0626 \\
\hline & 26 & 0.0487 & 0.0510 & 0.0522 & 0.0538 & 0.0699 & 0.0732 & 0.0763 & 0.0832 & 0.0614 \\
\hline & 27 & 0.0477 & 0.0499 & 0.0512 & 0.0528 & 0.0691 & 0.0725 & 0.0758 & 0.0828 & 0.0605 \\
\hline & 28 & 0.0469 & 0.0492 & 0.0505 & 0.0521 & 0.0693 & 0.0729 & 0.0762 & 0.0837 & 0.0602 \\
\hline & 29 & 0.0465 & 0.0489 & 0.0503 & 0.0521 & 0.0710 & 0.0752 & 0.0792 & 0.0883 & 0.0609 \\
\hline & 30 & 0.0466 & 0.0495 & 0.0511 & 0.0531 & 0.0779 & 0.0842 & 0.0906 & 0.1068 & 0.0645 \\
\hline
\end{tabular}

Wu's approximated method. We consider $3 \leq k \leq r-3$ to ensure the variance existence of $F_{2(r-k), 2 k}$ and its symmetry $F_{2 k, 2(r-k)}$. We choose the true values of parameters $\beta=1$, $\theta=1$ and significance level $\alpha=0.05$ and calculate the interval estimations of parameter $\beta$ based on 1000 Monte-Carlo simulations. The average upper and lower limits, average interval widths, and coverage probabilities are shown in Table 3 , in which the least average interval width occurs at $k=$ $[r / 3]$, where $[\cdot]$ is the greatest integer function. Thus it seems that the shortest interval is achieved when the sampling distribution is close to symmetric as much as possible.

\section{Simulation Study}

We investigate the precisions of presented point and interval estimation methods by Monte-Carlo simulations. We choose the true values of parameters $\beta=1, \theta=1$, under which the density curve is shown in Figure 1(a), whose time scale is approximately 0 to 2 . We generate 1000 random samples for each case of $(n, r)$ in Table 4 . The range of sample values is somehow related to the settings of $(n, r)$. For example, the range of $r=8$ observed values is 0.051 to 0.981 under $n=10$, $r=8$, while they are 0.0284 to 1.362 for $n=20, r=18$, and 0.018 and 1.483 for $n=30, r=30$. The point estimates of parameters $\beta, \theta$ for the 1000 generated samples under each case of $(n, r)$ are calculated. Table 4 lists the mean and mean square error (MSE) of these estimates, among which MIM consistently produces the smallest MSE for the estimates and interestingly performs better than MLE under moderate sample size. To make more comparison, we also computed MOM estimates based on Wu's exact quantity by setting up the equations through the first moments of $2 S_{r} \sim \chi_{2 r}^{2},(k /(r-$ $k))\left(\left(S_{r}-S_{k}\right) / S_{k}\right) \sim F_{2(r-k), 2 k}, k=1,2, \ldots, r-1$. The least MSE occurs at $k=[r / 3]$ as well, and it lies (not shown in the table) between MSEs of MMOM and IMOM, so is larger than MIM's. The $95 \%$ confidence intervals for $\beta$ are also computed based on these simulated samples. The average upper and lower limits, average interval width and coverage probability are shown in Table 5, where Wu's exact method in (12) selects the optimal interval estimation with $k=[r / 3]$. It can be seen that MIM quantity in (9) produces the shortest average width of interval as well as least MSE for point estimation. We also did simulation studies under various settings of parameter values, including the case of bathtub-shape failure rate with $0<\beta<1$. Similar results are obtained, showing that MIM approach consistently outperforms the other methods for both point and interval estimations (summary tables are not shown). The reason is that the log-scale expressed MIM quantity possesses less variability or its distribution is closer to symmetry than the distributions of other pivotal quantities. To illustrate this point, the density curves with $n=20, r=$ 16 of the four applied pivotal quantities: MOM, MIM, and 
TABLE 3: 95\% confidence interval for $\beta$ in 1000 simulations by Wu's exact.

\begin{tabular}{|c|c|c|c|c|c|c|}
\hline$n$ & $r$ & $k$ & $\begin{array}{c}\text { Average } \\
\text { Lower limit }\end{array}$ & $\begin{array}{c}\text { Average } \\
\text { Upper limit }\end{array}$ & $\begin{array}{c}\text { Average } \\
\text { Interval width }\end{array}$ & $\begin{array}{l}\text { Coverage } \\
\text { Probability }\end{array}$ \\
\hline \multirow{6}{*}{10} & \multirow{3}{*}{8} & 3 & 0.4016 & 2.6205 & 2.2189 & 0.956 \\
\hline & & 4 & 0.3626 & 2.7726 & 2.4100 & 0.951 \\
\hline & & 5 & 0.3293 & 3.3279 & 2.9986 & 0.961 \\
\hline & \multirow{3}{*}{9} & 3 & 0.4493 & 2.3191 & 1.8699 & 0.953 \\
\hline & & 4 & 0.4183 & 2.3419 & 1.9237 & 0,951 \\
\hline & & 5 & 0.4190 & 2.7233 & 2.3043 & 0.942 \\
\hline \multirow{6}{*}{15} & \multirow{3}{*}{13} & 3 & 0.5218 & 2.0465 & 1.5247 & 0.951 \\
\hline & & 4 & 0.5113 & 2.0149 & 1.5036 & 0.946 \\
\hline & & 5 & 0.5113 & 2.0591 & 1.5478 & 0.945 \\
\hline & \multirow{3}{*}{14} & 3 & 0.5350 & 1.9170 & 1.3820 & 0.951 \\
\hline & & 4 & 0.5267 & 1.8683 & 1.3415 & 0.956 \\
\hline & & 5 & 0.5238 & 1.8768 & 1.3530 & 0.968 \\
\hline \multirow{12}{*}{20} & \multirow{4}{*}{16} & 4 & 0.5443 & 1.9095 & 1.3652 & 0.951 \\
\hline & & 5 & 0.5324 & 1.8901 & 1.3577 & 0.956 \\
\hline & & 6 & 0.5350 & 1.9469 & 1.4120 & 0.954 \\
\hline & & 7 & 0.5248 & 1.9774 & 1.4526 & 0.954 \\
\hline & \multirow{4}{*}{17} & 4 & 0.5576 & 1.8412 & 1.2836 & 0.955 \\
\hline & & 5 & 0.5490 & 1.8199 & 1.2709 & 0.951 \\
\hline & & 6 & 0.5599 & 1.8739 & 1.3140 & 0.953 \\
\hline & & 7 & 0.5420 & 1.8670 & 1.3250 & 0.962 \\
\hline & \multirow{4}{*}{18} & 5 & 0.5759 & 1.7837 & 1.2078 & 0.945 \\
\hline & & 6 & 0.5658 & 1.7607 & 1.1948 & 0.956 \\
\hline & & 7 & 0.5673 & 1.7889 & 1.2216 & 0.943 \\
\hline & & 8 & 0.5615 & 1.8075 & 1.2460 & 0.957 \\
\hline \multirow{12}{*}{25} & \multirow{4}{*}{20} & 5 & 0.5833 & 1.7904 & 1.2071 & 0.951 \\
\hline & & 6 & 0.5726 & 1.7644 & 1.1918 & 0.942 \\
\hline & & 7 & 0.5838 & 1.8197 & 1.2359 & 0.951 \\
\hline & & 8 & 0.5695 & 1.8198 & 1.2503 & 0.962 \\
\hline & \multirow{4}{*}{21} & 6 & 0.5934 & 1.7386 & 1.1452 & 0.944 \\
\hline & & 7 & 0.5854 & 1.7279 & 1.1425 & 0.946 \\
\hline & & 8 & 0.5749 & 1.7237 & 1.1488 & 0.950 \\
\hline & & 9 & 0.5810 & 1.7754 & 1.1944 & 0.951 \\
\hline & \multirow{4}{*}{22} & 6 & 0.6034 & 1.6876 & 1.0842 & 0.951 \\
\hline & & 7 & 0.5974 & 1.6753 & 1.0779 & 0.949 \\
\hline & & 8 & 0.5910 & 1.6730 & 1.0820 & 0.949 \\
\hline & & 9 & 0.5925 & 1.6984 & 1.1060 & 0.947 \\
\hline \multirow{12}{*}{30} & \multirow{4}{*}{25} & 7 & 0.6201 & 1.6580 & 1.0378 & 0.952 \\
\hline & & 8 & 0.6097 & 1.6447 & 1.0350 & 0.946 \\
\hline & & 9 & 0.6080 & 1.6520 & 1.0439 & 0.950 \\
\hline & & 10 & 0.6084 & 1.6730 & 1.0646 & 0.954 \\
\hline & \multirow{4}{*}{26} & 7 & 0.6301 & 1.6321 & 1.0020 & 0.944 \\
\hline & & 8 & 0.6219 & 1.6100 & 0.9881 & 0.951 \\
\hline & & 9 & 0.6193 & 1.6158 & 0.9965 & 0.946 \\
\hline & & 10 & 0.6166 & 1.6181 & 1.0016 & 0.958 \\
\hline & \multirow{4}{*}{27} & 8 & 0.6389 & 1.5909 & 0.9520 & 0.945 \\
\hline & & 9 & 0.6303 & 1.5768 & 0.9465 & 0.955 \\
\hline & & 10 & 0.6324 & 1.5931 & 0.9607 & 0.948 \\
\hline & & 11 & 0.6298 & 1.5955 & 0.9657 & 0.947 \\
\hline
\end{tabular}


TABle 4: Comparison of point estimations for $\beta$ (above), $\theta$ (below) with true values $\beta=\theta=1$.

\begin{tabular}{|c|c|c|c|c|c|c|c|c|c|c|c|}
\hline \multirow{2}{*}{$n$} & \multirow{2}{*}{$r$} & \multicolumn{2}{|c|}{ MLE } & \multicolumn{2}{|c|}{ MOM } & \multicolumn{2}{|c|}{ MMOM } & \multicolumn{2}{|c|}{ IMOM } & \multicolumn{2}{|c|}{ MIM } \\
\hline & & Mean & MSE & Mean & MSE & Mean & MSE & Mean & MSE & Mean & MSE \\
\hline \multirow{6}{*}{10} & \multirow{2}{*}{8} & 1.3251 & 0.4554 & 1.5849 & 1.0819 & 1.2588 & 0.4836 & 1.1701 & 0.3111 & 1.0034 & 0.2089 \\
\hline & & 0.8921 & 0.1787 & 0.7928 & 0.2181 & 0.9077 & 0.1686 & 0.9427 & 0.1575 & 1.0073 & 0.1378 \\
\hline & \multirow{2}{*}{9} & 1.2712 & 0.3197 & 1.4863 & 0.7610 & 1.2325 & 0.3735 & 1.1225 & 0.2122 & 0.9882 & 0.1534 \\
\hline & & 0.9241 & 0.1555 & 0.8650 & 0.1889 & 0.9260 & 0.1477 & 0.9604 & 0.1452 & 0.9948 & 0.1214 \\
\hline & \multirow{2}{*}{10} & 1.1985 & 0.1949 & 1.3882 & 0.5076 & 1.1983 & 0.2675 & 1.0959 & 0.1413 & 0.9873 & 0.1082 \\
\hline & & 0.9933 & 0.1416 & 0.9883 & 0.1848 & 0.9879 & 0.1493 & 1.0005 & 0.1231 & 1.0088 & 0.1019 \\
\hline & \multirow{2}{*}{12} & 1.1955 & 0.1955 & 1.3323 & 0.3511 & 1.1466 & 0.1862 & 1.0893 & 0.1334 & 0.9907 & 0.1037 \\
\hline \multirow{7}{*}{15} & & 0.9197 & 0.1146 & 0.8448 & 0.1350 & 0.9305 & 0.1090 & 0.9704 & 0.1023 & 1.0107 & 0.0937 \\
\hline & \multirow{2}{*}{13} & 1.1565 & 0.1576 & 1.2694 & 0.2795 & 1.1167 & 0.1622 & 1.0744 & 0.1209 & 0.9878 & 0.0975 \\
\hline & & 0.9515 & 0.1028 & 0.9002 & 0.1180 & 0.9567 & 0.0984 & 0.9746 & 0.0929 & 1.0016 & 0.0837 \\
\hline & \multirow{2}{*}{14} & 1.1349 & 0.1215 & 1.2318 & 0.2377 & 1.1073 & 0.1404 & 1.0650 & 0.0798 & 0.9887 & 0.0655 \\
\hline & & 0.9629 & 0.0963 & 0.9346 & 0.1099 & 0.9642 & 0.0931 & 0.9784 & 0.0793 & 0.9944 & 0.0704 \\
\hline & \multirow{2}{*}{15} & 1.1200 & 0.0923 & 1.2292 & 0.2030 & 1.1257 & 0.1316 & 1.0525 & 0.0682 & 0.9858 & 0.0579 \\
\hline & & 0.9853 & 0.0867 & 0.9814 & 0.1068 & 0.9832 & 0.0891 & 0.9906 & 0.0808 & 0.9960 & 0.0715 \\
\hline \multirow{8}{*}{20} & \multirow{2}{*}{17} & 1.1305 & 0.1007 & 1.2089 & 0.1668 & 1.0958 & 0.1048 & 1.0673 & 0.0804 & 1.0023 & 0.0669 \\
\hline & & 0.9503 & 0.0732 & 0.9069 & 0.0836 & 0.9569 & 0.0716 & 0.9717 & 0.0676 & 0.9940 & 0.0625 \\
\hline & \multirow{2}{*}{18} & 1.1028 & 0.0834 & 1.1752 & 0.1409 & 1.0781 & 0.0938 & 1.0492 & 0.0601 & 0.9905 & 0.0516 \\
\hline & & 0.9621 & 0.0702 & 0.9287 & 0.0785 & 0.9629 & 0.0686 & 0.9836 & 0.0662 & 0.9991 & 0.0608 \\
\hline & \multirow{2}{*}{19} & 1.0948 & 0.0682 & 1.1685 & 0.1215 & 1.0867 & 0.0841 & 1.0450 & 0.0524 & 0.9915 & 0.0456 \\
\hline & & 0.9716 & 0.0575 & 0.9502 & 0.0659 & 0.9691 & 0.0581 & 0.9857 & 0.0637 & 0.9953 & 0.0582 \\
\hline & \multirow{2}{*}{20} & 1.0921 & 0.0599 & 1.1683 & 0.1197 & 1.0978 & 0.0854 & 1.0371 & 0.0519 & 0.9892 & 0.0463 \\
\hline & & 0.9974 & 0.0614 & 0.9938 & 0.0707 & 0.9954 & 0.0625 & 1.0030 & 0.0574 & 1.0061 & 0.0525 \\
\hline \multirow{8}{*}{25} & \multirow{2}{*}{22} & 1.0801 & 0.0626 & 1.1397 & 0.0979 & 1.0597 & 0.0692 & 1.0399 & 0.0528 & 0.9925 & 0.0468 \\
\hline & & 0.9624 & 0.0508 & 0.9294 & 0.0576 & 0.9631 & 0.0508 & 0.9824 & 0.0495 & 0.9968 & 0.0463 \\
\hline & 23 & 1.0782 & 0.0524 & 1.1362 & 0.0852 & 1.0644 & 0.0607 & 1.0358 & 0.0459 & 0.9917 & 0.0411 \\
\hline & & 0.9746 & 0.0499 & 0.9507 & 0.0561 & 0.9741 & 0.0502 & 0.9848 & 0.0468 & 0.9952 & 0.0437 \\
\hline & 24 & 1.0789 & 0.0523 & 1.1273 & 0.0823 & 1.0656 & 0.0609 & 1.0489 & 0.0454 & 1.0075 & 0.0398 \\
\hline & 24 & 0.9795 & 0.0486 & 0.9649 & 0.0531 & 0.9781 & 0.0480 & 0.9876 & 0.0463 & 0.9940 & 0.0429 \\
\hline & 25 & 1.0634 & 0.0405 & 1.1251 & 0.0754 & 1.0725 & 0.0572 & 1.0267 & 0.0397 & 0.9893 & 0.0366 \\
\hline & 25 & 1.0019 & 0.0464 & 0.9994 & 0.0531 & 1.0007 & 0.0481 & 1.0136 & 0.0423 & 1.0158 & $\mathbf{0 . 0 3 9 5}$ \\
\hline & 27 & 1.0679 & 0.0445 & 1.1110 & 0.0683 & 1.0494 & 0.0508 & 1.0310 & 0.0387 & 0.9934 & 0.0351 \\
\hline & & 0.9775 & 0.0449 & 0.9536 & 0.0483 & 0.9775 & 0.0441 & 0.9831 & 0.0409 & 0.9933 & 0.0387 \\
\hline & 28 & 1.0649 & 0.0399 & 1.1101 & 0.0617 & 1.0556 & 0.0467 & 1.0293 & 0.0342 & 0.9939 & 0.0312 \\
\hline 30 & & 0.9755 & 0.0408 & 0.9590 & 0.0451 & 0.9756 & 0.0412 & 0.9846 & 0.0389 & 0.9922 & 0.0367 \\
\hline & 29 & 1.0606 & 0.0379 & 1.0957 & 0.0572 & 1.0492 & 0.0451 & 1.0386 & 0.0352 & 1.0052 & 0.0317 \\
\hline & 29 & 0.9845 & 0.0404 & 0.9746 & 0.0425 & 0.9839 & 0.0395 & 0.9860 & 0.0370 & 0.9909 & 0.0349 \\
\hline & 30 & 1.0549 & 0.0315 & 1.1104 & 0.0593 & 1.0677 & 0.0464 & 1.0159 & 0.0298 & 0.9853 & 0.0282 \\
\hline & & 1.0014 & 0.0373 & 0.9980 & 0.0421 & 0.9992 & 0.0389 & 1.0046 & 0.0368 & 1.0065 & 0.0348 \\
\hline
\end{tabular}

Wu's exact and approx. were shown in Figure 5, where the skewnesses of these distributions are 1.0878, 0.5164, 3.5058, and 3.5156, respectively, indicating the distribution of MIM pivot (whose skewness is 0.5164 ) is closest to symmetry.

\section{Illustrative Examples}

In this section, we apply the presented approaches on two examples to compare the efficiency of estimation methods.
Example 1. Wu et al. [11] presented a complete failure hour of 18 electronic equipments: 5, 11, 21, 31, 46, 75, 98, 122, 145, $165,195,224,245,293,321,330,350$, and 420. Xie et al. [18] showed that the data had bathtub failure rate function, so we fit this dataset by $\operatorname{CZM}(\beta, \theta)$ to obtain the estimation results summarized in Table 6 . In this case of relatively small sample size $n=18$, MIM should yield reliable estimates based on the simulation study. From the results of the $95 \%$ confidence interval for $\beta$, one may see that the shortest Wu's exact 
TABLE 5: Width comparison of interval estimations for 1000 simulations.

\begin{tabular}{|c|c|c|c|c|}
\hline$n$ & $r$ & $\begin{array}{c}\text { Wu's exact } \\
\text { (coverage probability) }\end{array}$ & $\begin{array}{c}\text { MOM pivot } \\
\text { (coverage probability) }\end{array}$ & $\begin{array}{c}\text { MIM pivot } \\
\text { (coverage probability) }\end{array}$ \\
\hline \multirow{3}{*}{10} & 8 & $2.2189(0.956)$ & $2.4262(0.962)$ & $\mathbf{1 . 6 8 5 0}(0.951)$ \\
\hline & 9 & $1.8699(0.953)$ & $2.0865(0.960)$ & $1.4650(0.954)$ \\
\hline & 10 & $1.5398(0.954)$ & $1.8407(0.957)$ & $1.2372(0.952)$ \\
\hline \multirow{4}{*}{15} & 12 & $1.5989(0.946)$ & $1.6231(0.958)$ & $1.2542(0.956)$ \\
\hline & 13 & $1.5036(0.946)$ & $1.5421(0.946)$ & $1.1714(0.955)$ \\
\hline & 14 & $1.3415(0.956)$ & $1.3138(0.949)$ & $\mathbf{1 . 0 6 0 1}(0.953)$ \\
\hline & 15 & $1.1966(0.956)$ & $1.2606(0.951)$ & $\mathbf{0 . 9 5 3 7}(0.949)$ \\
\hline \multirow{5}{*}{20} & 16 & $1.3577(0.956)$ & $1.2771(0.952)$ & $1.0681(0.950)$ \\
\hline & 17 & $1.2709(0.951)$ & $1.2150(0.961)$ & $1.0018(0.963)$ \\
\hline & 18 & $1.1948(0.956)$ & $1.1268(0.953)$ & $\mathbf{0 . 9 3 6 0}(0.955)$ \\
\hline & 19 & $1.1022(0.950)$ & $1.0185(0.943)$ & $\mathbf{0 . 8 8 2 1}(0.960)$ \\
\hline & 20 & $1.0075(0.950)$ & $1.0016(0.947)$ & $\mathbf{0 . 8 1 0 0}(0.950)$ \\
\hline \multirow{6}{*}{25} & 20 & $1.1918(0.942)$ & $1.0922(0.943)$ & $0.9306(0.948)$ \\
\hline & 21 & $1.1425(0.946)$ & $1.0411(0.959)$ & $\mathbf{0 . 8 8 9 6}(0.950)$ \\
\hline & 22 & $1.0779(0.949)$ & $0.9787(0.954)$ & $\mathbf{0 . 8 4 5 5}(0.953)$ \\
\hline & 23 & $1.0166(0.945)$ & $0.9380(0.954)$ & $\mathbf{0 . 8 0 2 5}(0.955)$ \\
\hline & 24 & $0.9526(0.946)$ & $0.8832(0.948)$ & $\mathbf{0 . 7 5 7 8}(0.960)$ \\
\hline & 25 & $0.8863(0.951)$ & $0.8644(0.943)$ & $\mathbf{0 . 7 1 4 9}(0.953)$ \\
\hline \multirow{7}{*}{30} & 24 & $1.0806(0.950)$ & $0.9752(0.954)$ & $\mathbf{0 . 8 4 2 0}(0.949)$ \\
\hline & 25 & $1.0350(0.946)$ & $0.9187(0.945)$ & $\mathbf{0 . 8 0 8 6}(0.956)$ \\
\hline & 26 & $0.9881(0.951)$ & $0.8959(0.951)$ & $\mathbf{0 . 7 7 8 5}(0.956)$ \\
\hline & 27 & $0.9465(0.955)$ & $0.8407(0.955)$ & $\mathbf{0 . 7 4 5 4}(0.961)$ \\
\hline & 28 & $0.9022(0.956)$ & $0.8212(0.945)$ & $\mathbf{0 . 7 1 5 0}(0.951)$ \\
\hline & 29 & $0.8543(0.943)$ & $0.7807(0.941)$ & $\mathbf{0 . 6 7 9 2}(0.954)$ \\
\hline & 30 & $0.8039(0.955)$ & $0.7714(0.947)$ & $\mathbf{0 . 6 4 3 8}(0.948)$ \\
\hline
\end{tabular}

interval occurs at $k=7$, a little narrower than that of Wu's exact with $k=[18 / 3]=6$. This indicates that maybe the data slightly deviate from $\operatorname{CZM}(\beta, \theta)$. Although Wu's approx. gives a shorter width than that from Wu's exact, it is a large-sample based approach whose coverage probability might not be the same as the nominal under the small sample size. Overall, MIM pivot produces the shortest interval among all the methods in accordance with the findings in the simulation study.

Example 2. Chen [6] presented a type-II censoring data of $n=15$ samples from CZM $(0.5,50)$, in which there was $r=11$ complete failures: $0.29,1.44,8.38,8.66,10.20,11.04,13.44$, $14.37,17.05,17.13$, and 18.35. Notably, since $\beta=0.5<1$, the data was from the distribution with a bathtub-shaped failure rate. The estimation results by the proposed methods are shown in Table 7, where we notice that some methods perform better than MIM (particularly for the estimation of $\beta$ ), but not significantly. Despite only 11 complete failures out of 15 samples used in the estimation, MIM still yields a comparable point estimates. For the $95 \%$ confidence interval of $\beta$, Wu's exact and approx. with $k=[11 / 3]=3$ produce the least lengths among all intervals for $k=1,2, \ldots, 10$. Also MIM pivot gives the shortest length of interval as expected. In summary, although it is an example of bathtub-shaped failure rate, the results are in accordance with the findings in the simulation study, where the comparisons are based on the data generated from a nonbathtub shaped failure rate function with $\beta=1$.

\section{Conclusions}

In this paper, we consider the parameter inference for a twoparameter life distribution. We present five point estimations and three interval estimations for the parameter of interest. Through the Monte-Carlo simulation technique, we determined a mechanism to obtain the optimal interval estimation originally proposed by $\mathrm{Wu}$ et al. [11]. The performance of the methods were assessed by a comparison of estimation precisions, and it showed that the MIM pivotal quantity with a log-scale expression leads to a relative effectiveness of the estimation under moderate sample size. This indicated that the quantity has less variability or its distribution is closer to symmetry. We applied the methods to two datasets and demonstrated the efficiency of MIM estimation method.

\section{Appendix}

Proof of Lemma 1. (1) When $\beta=1$, we have $f^{\prime}(x)=(1-$ $\left.e^{x} / \theta\right) / \theta \exp \left(x+\left(1-e^{x}\right) / \theta\right)$. Obviously, $f^{\prime}(x)<0$ when $0<$ $\theta \leq 1$, and $f(x)$ is unimodal at $x_{0}=\log \theta$ when $\theta>1$. 

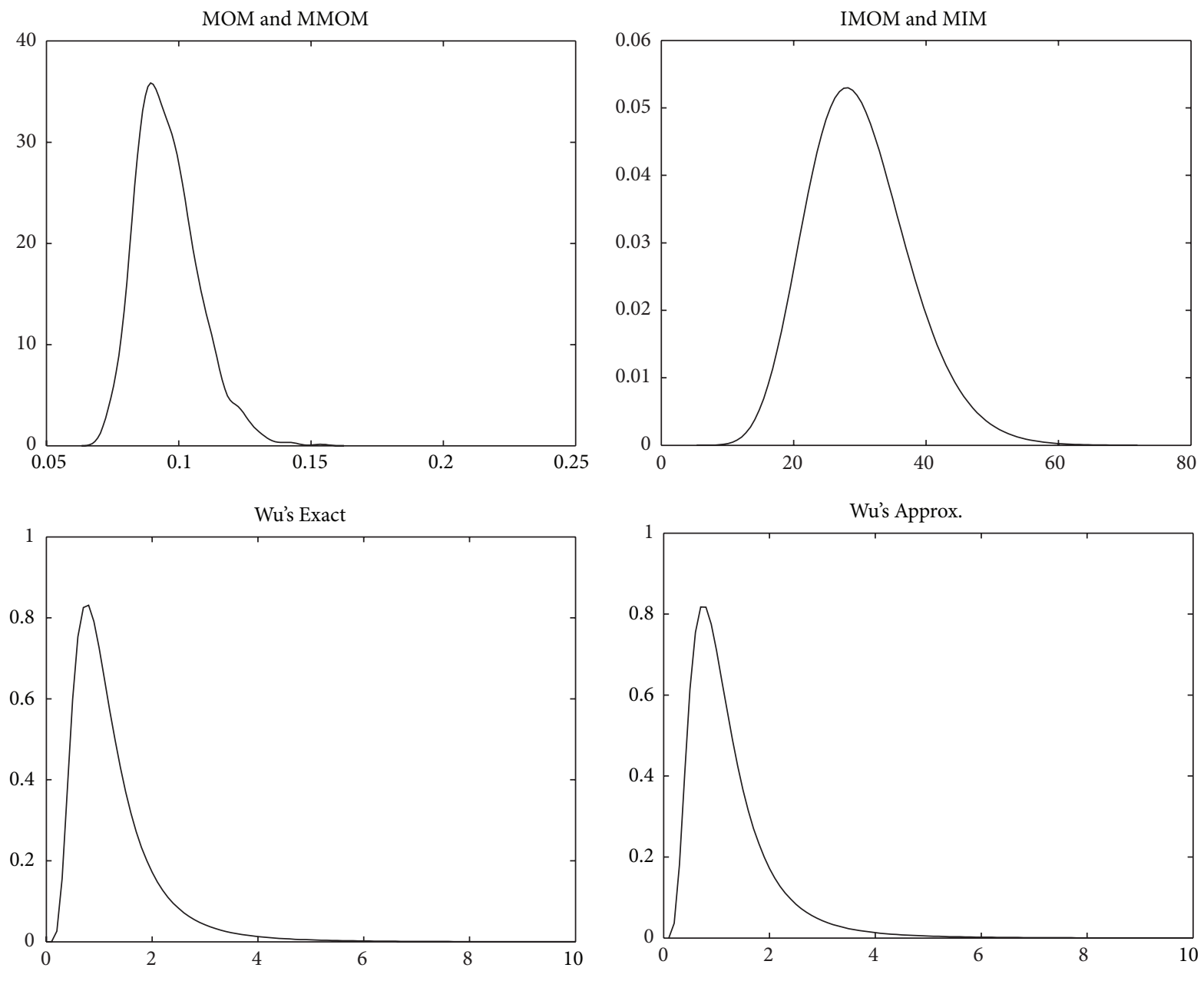

FIgURE 5: Densities of pivotal quantities with $n=20, r=16$.

TABLE 6: Estimation results using data in Wu et al. [11].

\begin{tabular}{|c|c|c|c|c|c|}
\hline Parameter & MLE & MOM & MMOM & IMOM & MIM \\
\hline$\beta$ & 0.2893 & 0.3102 & 0.3005 & 0.2806 & 0.2741 \\
\hline$\theta$ & 100.4048 & 189.7327 & 139.5206 & 78.9727 & 66.6931 \\
\hline \multicolumn{2}{|c|}{ Approach } & \multicolumn{3}{|c|}{$95 \%$ confidence interval for $\beta$} & Length \\
\hline \multicolumn{2}{|c|}{ Chen's (Wu's exact $k=1$ ) } & \multicolumn{3}{|c|}{$(0.1986,0.3697)$} & 0.1771 \\
\hline \multicolumn{2}{|c|}{ Wu's exact $(k=7)$} & \multicolumn{3}{|c|}{$(0.2106,0.3299)$} & 0.1193 \\
\hline \multicolumn{2}{|c|}{ Wu's exact $(k=6)$} & \multicolumn{3}{|c|}{$(0.2066,0.3261)$} & 0.1195 \\
\hline \multicolumn{2}{|c|}{ Wu's approximated $(k=5)$} & \multicolumn{3}{|c|}{$(0.2122,0.3220)$} & 0.1098 \\
\hline \multicolumn{2}{|c|}{ MOM pivot } & \multicolumn{3}{|c|}{$(0.2304,0.3640)$} & 0.1336 \\
\hline \multicolumn{2}{|c|}{ MIM pivot } & \multicolumn{3}{|c|}{$(0.2237,0.3260)$} & 0.1023 \\
\hline
\end{tabular}

For $\beta \neq 1$, first we have

$$
\begin{aligned}
f^{\prime}(x)= & \frac{\beta}{\theta^{2}} x^{\beta-2}\left[(\beta-1) \theta+\beta \theta x^{\beta}-\beta x^{\beta} e^{x^{\beta}}\right] \\
& \times \exp \left(x^{\beta}+\frac{1-e^{x^{\beta}}}{\theta}\right) .
\end{aligned}
$$

Let $g(x)=(\beta-1) \theta+\beta \theta x^{\beta}-\beta x^{\beta} e^{x^{\beta}}$, then obviously $\lim _{x \rightarrow 0} g(x)=(\beta-1) \theta, \lim _{x \rightarrow+\infty} g(x)=-\infty$, and $g^{\prime}(x)=$ $\beta^{2} x^{\beta-1}\left[\theta-e^{x^{\beta}}-x^{\beta} e^{x^{\beta}}\right]$. Let $g_{1}(x)=\theta-e^{x^{\beta}}-x^{\beta} e^{x^{\beta}}$, then $g_{1}^{\prime}(x)<0$ and $\lim _{x \rightarrow 0} g_{1}(x)=\theta-1, \lim _{x \rightarrow+\infty} g_{1}(x)=-\infty$. (i) for $0<\theta \leq 1$, we have $g_{1}(x)<0$, and thus $g^{\prime}(x)<0$. (ii) for $\theta>1$, there is a unique positive $\operatorname{root} x_{1}$ of $g_{1}(x)=0$, 
TABLE 7: Estimation results using data in Chen [6].

\begin{tabular}{|c|c|c|c|c|c|}
\hline Parameter & MLE & MOM & MMOM & IMOM & MIM \\
\hline$\beta$ & 0.5068 & 0.5471 & 0.5071 & 0.4648 & 0.4584 \\
\hline$\theta$ & 63.8939 & 102.2165 & 64.7495 & 40.3122 & 41.5428 \\
\hline \multicolumn{2}{|c|}{ Approach } & \multicolumn{3}{|c|}{$95 \%$ confidence interval for $\beta$} & Length \\
\hline \multicolumn{2}{|c|}{ Chen's (Wu's exact $k=1$ ) } & \multicolumn{3}{|c|}{$(0.1900,0.6200)$} & 0.4300 \\
\hline \multicolumn{2}{|c|}{ Wu's exact $(k=3)$} & \multicolumn{3}{|c|}{$(0.3783,0.7049)$} & 0.3266 \\
\hline \multicolumn{2}{|c|}{ Wu's approximated $(k=3)$} & \multicolumn{3}{|c|}{$(0.3803,0.6957)$} & 0.3154 \\
\hline \multicolumn{2}{|c|}{ MOM pivot } & \multicolumn{3}{|c|}{$(0.3013,0.6492)$} & 0.3479 \\
\hline \multicolumn{2}{|c|}{ MIM pivot } & \multicolumn{3}{|c|}{$(0.2955,0.5998)$} & 0.3043 \\
\hline
\end{tabular}

resulting in $g_{1}(x)>0, g^{\prime}(x)>0$ in $0<x<x_{1}$, and $g_{1}(x)<0, g^{\prime}(x)<0$ in $x>x_{1}$.

(2) When $\beta>1$, we know $\lim _{x \rightarrow 0} g(x)=(\beta-1) \theta>0$, so there exists a unique positive root $x_{0}$ of $g(x)=0$. Therefore, $g(x)>0$, and then $f^{\prime}(x)>0$ in $0<x<x_{0}$, but $g(x)<0$, then $f^{\prime}(x)<0$ in $x>x_{0}$, as a result $f(x)$ is unimodal.

(3) When $0<\beta<1$, then $\lim _{x \rightarrow 0} g(x)=(\beta-1) \theta<$ $0, \lim _{x \rightarrow+\infty} g(x)=-\infty$. Note that $\lim _{x \rightarrow 0} f(x)=+\infty$, $\lim _{x \rightarrow+\infty} f(x)=0$. (i) for $0<\theta \leq 1$, then we have $g^{\prime}(x)<0$, and so $g(x)<0$, thus $f^{\prime}(x)<0$, that is, $f(x)$ is decreasing; (ii) for $\theta>1$, (a) if $g\left(x_{1}\right) \leq 0$, then we have $g(x) \leq 0, f^{\prime}(x) \leq 0$, that is, $f(x)$ is decreasing; (b) if $g\left(x_{1}\right)>0$, then there are two positive roots $x_{01}, x_{02}$ of $g(x)=0$ with $0<x_{01}<x_{1}<x_{02}$. First for $0<x<x_{01}$, we have $g(x)<0, f^{\prime}(x)<0$, and so $f(x)$ is decreasing. Secondly for $x_{01}<x<x_{02}$, we have $g(x)>0, f^{\prime}(x)>0$, and so $f(x)$ is increasing. Finally for $x>x_{02}$, we have $g(x)<0, f^{\prime}(x)<0$, and so $f(x)$ is decreasing.

The following mathematical result will be used in the proof of Theorem 4 .

Lemma A.1 (see [19]). Let $g(x)$ be a nonnegative function at $[a,+\infty)$ and integrable at $[a, b]$ for any $b>a$. If $\lim _{x \rightarrow+\infty}((\log g(x)) / \log x)=p$, then $\int_{a}^{+\infty} g(x) d x$ is convergent for $-\infty \leq p<-1$, and divergent for $-1<p \leq+\infty$.

Proof of Theorem 4. (1) The $k$ th moment is

$$
E X^{k}=\frac{\beta}{\theta} \int_{0}^{+\infty} x^{k+\beta-1} \exp \left(x^{\beta}+\frac{1-e^{x^{\beta}}}{\theta}\right) d x .
$$

Let the integrand function $g(x)=x^{k+\beta-1} \exp \left(x^{\beta}+\left(1-e^{x^{\beta}}\right) / \theta\right)$ and then by L'Hopital's rule with $t=x^{\beta}$

$$
\begin{aligned}
\lim _{x \rightarrow+\infty} \frac{\log g(x)}{\log x} \\
=\frac{1}{\theta} \lim _{t \rightarrow+\infty} \frac{\theta(k+\beta-1) \log t+\beta \theta t+\beta-\beta e^{t}}{\log t} \\
=\frac{1}{\theta} \lim _{t \rightarrow+\infty}\left[\theta(k+\beta-1)+\beta \theta t-\beta t e^{t}\right]=-\infty .
\end{aligned}
$$

Thus the $k$ th moment exists by Lemma A.1.
(2) $G(x)=-(\log (1-F(x))) / x=\left(e^{x^{\beta}}-1\right) / \theta x$, so $G^{\prime}(x)=$ $\left(1 / \theta x^{2}\right)\left(\beta x^{\beta} e^{x^{\beta}}-e^{x^{\beta}}+1\right)$. Setting the function $g(x)=\beta x^{\beta} e^{x^{\beta}}-$ $e^{x^{\beta}}+1$, we have $\lim _{x \rightarrow 0} g(x)=0, \lim _{x \rightarrow+\infty} g(x)=+\infty$, and $g^{\prime}(x)=\beta^{2} x^{\beta-1} e^{x^{\beta}}\left(x^{\beta}-(1-\beta) / \beta\right)$, then

(i) when $\beta \geq 1$, we have $g^{\prime}(x)>0$, then $g(x)>0$, $G^{\prime}(x)>0$, that is, $G(x)$ is increasing;

(ii) when $0<\beta<1$, let $x_{0}=((1-\beta) / \beta)^{1 / \beta}$, and then we have $g^{\prime}(x)<0$ in $0<x<x_{0}$ and $g^{\prime}(x)>0$ in $x>x_{0}$. Also $g\left(x_{0}\right)=1-\beta e^{(1-\beta) / \beta}<0$, therefore, there exists a root $x_{1}$ of $g(x)=0$ with $x_{1}>x_{0}>0$. Then we have $g(x)<0, G^{\prime}(x)<0$, so $G(x)$ is decreasing in $0<x<x_{1}$, and $g(x)>0, G^{\prime}(x)>0$, so $G(x)$ is increasing in $x>x_{1}$. That is $G(x)$ is a bathtub-shape (concave-up) function.

(3) $m(x)=\left(\int_{x}^{+\infty}[1-F(t)] d t\right) /(1-F(x))=\left(\int_{x}^{+\infty} \exp ((1-\right.$ $\left.\left.\left.e^{t^{\beta}}\right) / \theta\right) d t\right) /\left(\exp \left(\left(1-e^{x^{\beta}}\right) / \theta\right)\right)$, then

$$
\begin{aligned}
m^{\prime}(x)= & \frac{\beta x^{\beta-1} e^{x^{\beta}}}{\theta \exp \left(\left(1-e^{x^{\beta}}\right) / \theta\right)} \\
& \times\left[\int_{x}^{+\infty} \exp \left(\frac{1-e^{t^{\beta}}}{\theta}\right) d t\right. \\
& \left.\quad-\frac{\theta}{\beta} x^{1-\beta} e^{-x^{\beta}} \exp \left(\frac{1-e^{x^{\beta}}}{\theta}\right)\right] .
\end{aligned}
$$

Let $g(x)=\int_{x}^{+\infty} \exp \left(\left(1-e^{t^{\beta}}\right) / \theta\right) d t-(\theta / \beta) x^{1-\beta} e^{-x^{\beta}} \exp ((1-$ $\left.\left.e^{x^{\beta}}\right) / \theta\right)$, then

$$
g^{\prime}(x)=\theta x^{-\beta} e^{-x^{\beta}}\left(x^{\beta}-\frac{1-\beta}{\beta}\right) \exp \left(\frac{1-e^{x^{\beta}}}{\theta}\right) .
$$

(i) When $\beta>1$, we have $g^{\prime}(x)>0, \lim _{x \rightarrow 0} g(x)=-\infty$, $\lim _{x \rightarrow+\infty} g(x)=0$, so $g(x)<0$, and thus $m^{\prime}(x)<0$.

(ii) When $\beta=1$, we have $g^{\prime}(x)=\theta e^{-x} \exp \left(\left(1-e^{x}\right) / \theta\right)>$ $0, \lim _{x \rightarrow 0} g(x)=\int_{0}^{+\infty} \exp \left(\left(1-e^{t}\right) / \theta\right) d t-\theta<0$, $\lim _{x \rightarrow+\infty} g(x)=0$, so $g(x)<0, m^{\prime}(x)<0$. 
(iii) When $0<\beta<1$, with $x_{0}=((1-\beta) / \beta)^{1 / \beta}$, we get $g^{\prime}(x)<0$ in $0<x<x_{0}$ and $g^{\prime}(x)>$ 0 in $x>x_{0}$. Also $\lim _{x \rightarrow 0} g(x)=\int_{0}^{+\infty} \exp ((1-$ $\left.\left.e^{t^{\beta}}\right) / \theta\right) d t>0, \lim _{x \rightarrow+\infty} g(x)=0$, and $g\left(x_{0}\right)=$ $\int_{x_{0}}^{+\infty} \exp \left(\left(1-e^{t^{\beta}}\right) / \theta\right) d t-(\theta / \beta) x_{0}^{1-\beta} e^{-x_{0}^{\beta}} \exp \left(\left(1-e^{x_{0}^{\beta}}\right) / \theta\right)$ $=(\theta / \beta) \int_{x_{0}}^{+\infty}\left[(1-\beta) t^{-\beta}-\beta\right] \exp \left(-t^{\beta}+\left(1-e^{t^{\beta}}\right) / \theta\right) d t<0$ since $(1-\beta) t^{-\beta}-\beta<0$ in $t>x_{0}$. Thus, there is a unique root $x_{1}$ of $g(x)=0$ with $0<x_{1}<x_{0}$, so that $g(x)>0, m^{\prime}(x)>0$ in $0<x<x_{1}$ and $g(x)<0$, $m^{\prime}(x)<0$ in $x>x_{1}$. That is, $m(x)$ is unimodal.

Proof of Lemma 5. The proof of (1) is clear, therefore we only show the proof for (2). Chen [6] gave a proof, but it was wrong since in general two increasing functions, say $f(x), g(x)$ can not guarantee the product $f(x) g(x)$ being increasing unless both $f(x)$ and $g(x)$ are positive functions. Here we give the correct proof as follows.

Since $\xi(\beta)=\exp \left\{\log \left(e^{b^{\beta}}-1\right)-\log \left(e^{a^{\beta}}-1\right)\right\}$, then

$$
\begin{aligned}
\xi^{\prime}(\beta)= & \exp \left\{\log \left(e^{b^{\beta}}-1\right)-\log \left(e^{a^{\beta}}-1\right)\right\} \\
& \times\left(\frac{b^{\beta} e^{b^{\beta}} \log b}{e^{b^{\beta}}-1}-\frac{a^{\beta} e^{a^{\beta}} \log a}{e^{a^{\beta}}-1}\right) .
\end{aligned}
$$

To show $\xi^{\prime}(\beta)>0$, it is desired to prove that the function $\delta(s)=\left(e^{s^{\beta}} s^{\beta} \log s\right) /\left(e^{s^{\beta}}-1\right)$ is strictly increasing in $s>$ 0 . To make the notation simple, let $x=s^{\beta}$, and $f(x)=$ $\left(x e^{x} \log x\right) /\left(e^{x}-1\right)=(x \log x) /\left(1-e^{-x}\right)$, we have $f^{\prime}(x)=$ $\left(e^{-x} /\left(1-e^{-x}\right)^{2}\right)\left[\left(e^{x}-1\right)+\left(e^{x}-1-x\right) \log x\right]$. Note that $e^{x}=$ $\sum_{k=0}^{\infty}\left(x^{k} / k !\right)$, then $g(x)=\left(e^{x}-1\right)+\left(e^{x}-1-x\right) \log x=$ $\sum_{k=1}^{\infty}\left(x^{k} /(k+1) !\right)(k+1+x \log x)$.

(i) for $x \geq 1$, obviously $\log x \geq 0$, and so $g(x)>0$. (ii) for $0<x<1$, first $k+1+x \log x \geq 2+x \log x$ for $k \geq 1$. Additionally, we know $g_{1}(x)=2+x \log x$ is a concaveup function in $0<x<1$ with achieving minimum value $g_{1}\left(e^{-1}\right)=2-e^{-1}>0$, so $g_{1}(x)>0$, and then $g(x)>0$ in $0<x<1$.

In conclusion, we obtain $g(x)>0$ in $x>0$, and so $f^{\prime}(x)>0$, that is, $f(x)$ is strictly increasing in $x>0$. The lemma follows.

Proof of Uniqueness of $M L E$. We denote the left side of the second equation in $(4)$ as $H(\beta)$. It is obvious that $\lim _{\beta \rightarrow 0} H(\beta)=-\infty$. Now we consider $\lim _{\beta \rightarrow+\infty} H(\beta)$ for various cases by using Lemma 5 .

(1) When $x_{(r)} \leq 1$, then $x_{(i)}<1, i=1,2, \ldots, r-1$. Note that

$H(\beta)$

$$
\begin{aligned}
= & \left(\left(\sum_{i=1}^{r-1} \frac{x_{(i)}^{\beta} e^{x_{(i)}^{\beta}} \log x_{(i)}}{e^{x_{(r)}^{\beta}}-1}+(n-r+1)\left(\frac{x_{(r)}^{\beta} e^{x_{(r)}^{\beta}} \log x_{(r)}}{e^{x_{(r)}^{\beta}}-1}\right)\right)\right. \\
& \left.\times\left(\sum_{i=1}^{r-1} \frac{e^{x_{(i)}^{\beta}}-1}{e^{x_{(r)}^{\beta}}-1}+(n-r+1)\right)^{-1}\right) \\
& -\frac{1}{r} \sum_{i=1}^{r} x_{(i)}^{\beta} \log x_{(i)}-\frac{1}{r} \sum_{i=1}^{r} \log x_{(i)}-\frac{1}{\beta}
\end{aligned}
$$

with $\lim _{\beta \rightarrow+\infty} x_{(i)}^{\beta}=0, \lim _{\beta \rightarrow+\infty}\left(\left(x_{(i)}^{\beta} e^{x_{(i)}^{\beta}} \log x_{(i)}\right) /\left(e^{x_{(r)}^{\beta}}-\right.\right.$ $1))=0, i<r, \lim _{\beta \rightarrow+\infty}\left(\left(x_{(r)}^{\beta} e^{x_{(r)}^{\beta}} \log x_{(r)}\right) /\left(e^{x_{(r)}^{\beta}}-1\right)\right)$ $=\lim _{\beta \rightarrow+\infty}\left(\left(\left(1+x_{(r)}^{\beta}\right) x_{(r)}^{\beta} e^{x_{(r)}^{\beta}} \log ^{2} x_{(r)}\right) /\left(x_{(r)}^{\beta} e^{x_{(r)}^{\beta}} \log x_{(r)}\right)\right)=$ $\log x_{(r)}$ and $\lim _{\beta \rightarrow+\infty} \sum_{i=1}^{r-1}\left(\left(e^{x_{(i)}^{\beta}}-1\right) /\left(e^{x_{(r)}^{\beta}}-1\right)\right)=0$ (since $\left.x_{(i)}<x_{(r)}\right)$, we have $\lim _{\beta \rightarrow+\infty} H(\beta)=\log x_{(r)}-$ $(1 / r) \sum_{i=1}^{r} \log x_{(i)}=(1 / r) \sum_{i=1}^{r-1}\left(\log x_{(r)}-\log x_{(i)}\right)>0$.

(2) When $x_{(r)}>1$, note that

$$
\begin{aligned}
H(\beta)= & x_{(r)}^{\beta}\left\{\frac{e^{x_{(r)}^{\beta}}}{e^{x_{(r)}^{\beta}-1}} \times \frac{\sum_{i=1}^{r-1}\left(x_{(i)} / x_{(r)}\right)^{\beta} e^{-x_{(r)}^{\beta}\left[1-\left(x_{(i)} / x_{(r)}\right)^{\beta}\right]} \log x_{(i)}+(n-r+1) \log x_{(r)}}{\sum_{i=1}^{r-1}\left(\left(e^{x_{(i)}^{\beta}}-1\right) /\left(e^{x_{(r)}^{\beta}}-1\right)\right)+(n-r+1)}-\frac{1}{r} \sum_{i=1}^{r}\left(\frac{x_{(i)}}{x_{(r)}}\right)^{\beta} \log x_{(i)}\right\} \\
& -\frac{1}{r} \sum_{i=1}^{r} \log x_{(i)}-\frac{1}{\beta}
\end{aligned}
$$

with $\lim _{\beta \rightarrow+\infty} x_{(r)}^{\beta}=+\infty, \lim _{\beta \rightarrow+\infty}\left(x_{(i)} / x_{(r)}\right)^{\beta}=0, i<$ $r, \lim _{\beta \rightarrow+\infty}\left(e^{x_{(r)}^{\beta}} /\left(e^{x_{(r)}^{\beta}}-1\right)\right)=1$, then $\lim _{\beta \rightarrow+\infty} H(\beta)=$ $\lim _{\beta \rightarrow+\infty} x_{(r)}^{\beta}\left(\log x_{(r)}-(1 / r) \log x_{(r)}\right)-(1 / r) \sum_{i=1}^{r} \log x_{(i)}=$ $+\infty$.

In conclusion, $H(\beta)$ is increasing and $\lim _{\beta \rightarrow 0} H(\beta)=$ $-\infty, \lim _{\beta \rightarrow+\infty} H(\beta)>0$, thus the equation $H(\beta)=0$ must have a unique positive root.
Proof of Uniqueness of MOM. Let $H(\beta)=\left(\sum_{i=1}^{r}\left(e^{x_{(i)}^{\beta}}-1\right)^{2}\right) /$ $\left[\sum_{i=1}^{r}\left(e^{x_{(i)}^{\beta}}-1\right)\right]^{2}$, then

$$
\begin{aligned}
H^{\prime}(\beta)= & \frac{1}{\left[\sum_{i=1}^{r}\left(e^{x_{(i)}^{\beta}}-1\right)\right]^{3}} \\
& \times \sum_{i=1}^{r} \sum_{j=1}^{r}\left[2\left(e^{x_{(i)}^{\beta}}-1\right)\left(e^{x_{(j)}^{\beta}}-1\right) x_{(i)}^{\beta} e^{x_{(i)}^{\beta}} \log x_{i}\right.
\end{aligned}
$$




$$
\begin{aligned}
& \left.-2\left(e^{x_{(i)}^{\beta}}-1\right)^{2} x_{(j)}^{\beta} e^{x_{(j)}^{\beta}} \log x_{(j)}\right] \\
& =\frac{1}{\left[\sum_{i=1}^{r}\left(e^{x_{(i)}^{\beta}}-1\right)\right]^{3}} \\
& \times \sum_{i=1}^{r} \sum_{j=1}^{r}\left[\left(e^{x_{(i)}^{\beta}}-1\right)\left(e^{x_{(j)}^{\beta}}-1\right) x_{(i)}^{\beta} e^{x_{(i)}^{\beta}} \log x_{(i)}\right. \\
& +\left(e^{x_{(i)}^{\beta}}-1\right)\left(e^{x_{(j)}^{\beta}}-1\right) x_{(j)}^{\beta} e^{x_{(j)}^{\beta}} \log x_{(j)} \\
& -\left(e^{x_{(i)}^{\beta}}-1\right)^{2} x_{(j)}^{\beta} e^{x_{(j)}^{\beta}} \log x_{(j)} \\
& \left.-\left(e^{x_{(j)}^{\beta}}-1\right)^{2} x_{(i)}^{\beta} e^{x_{(i)}^{\beta}} \log x_{(i)}\right] \\
& =\frac{1}{\left[\sum_{i=1}^{r}\left(e^{x_{(i)}^{\beta}}-1\right)\right]^{3}} \\
& \times \sum_{i=1}^{r} \sum_{j=1}^{r}\left(e^{x_{(i)}^{\beta}}-e^{x_{(j)}^{\beta}}\right) \\
& \times\left[\left(e^{x_{(j)}^{\beta}}-1\right) x_{(i)}^{\beta} e^{x_{(i)}^{\beta}} \log x_{(i)}\right. \\
& \left.-\left(e^{x_{(i)}^{\beta}}-1\right) x_{(j)}^{\beta} e^{x_{(j)}^{\beta}} \log x_{(j)}\right] \\
& =\frac{1}{\left[\sum_{i=1}^{r}\left(e^{x_{(i)}^{\beta}}-1\right)\right]^{3}} \\
& \times \sum_{i=1}^{r} \sum_{j=1}^{r}\left(e^{x_{(i)}^{\beta}}-1\right)\left(e^{x^{\beta}(j)}-1\right)\left(e^{x_{(i)}^{\beta}}-e^{x^{\beta}(j)}\right)^{2} \\
& \times\left(\left(\frac{x_{(i)}^{\beta} e^{x_{(i)}^{\beta}} \log x_{(i)}}{e^{x_{(i)}^{\beta}}-1}-\frac{x_{(j)}^{\beta} e^{x_{(j)}^{\beta}} \log x_{(j)}}{e^{x_{(j)}^{\beta}}-1}\right)\right. \\
& \left.\times\left(e^{x_{(i)}^{\beta}}-e^{x_{(j)}^{\beta}}\right)^{-1}\right) .
\end{aligned}
$$

Obviously, $e^{x^{\beta}}-1>0$ for $x>0$, and we would like to show the last function being positive in the above expression. Let $g_{1}(x)=e^{x^{\beta}}$ and $g_{2}(x)=\left(x^{\beta} e^{x^{\beta}} \log x\right) /\left(e^{x^{\beta}}-1\right)$, we have $g_{1}^{\prime}(x)=\beta x^{\beta-1} e^{x^{\beta}}>0$, and $g_{2}^{\prime}(x)>0$ from the proof of Lemma 5 . Then by the Mean Value Theorem, there are $\xi_{1}, \xi_{2}$ with $\min \left(x_{(i)}, x_{(j)}\right)<\xi_{1}, \xi_{2}<\max \left(x_{(i)}, x_{(j)}\right)$ satisfying

$$
\left(\frac{x_{(i)}^{\beta} e^{x_{(i)}^{\beta}} \log x_{(i)}}{e^{x_{(i)}^{\beta}}-1}-\frac{x_{(j)}^{\beta} e^{x_{(j)}^{\beta}} \log x_{(j)}}{e^{x_{(j)}^{\beta}}-1}\right)
$$

$$
\begin{aligned}
& \times\left(e^{x_{(i)}^{\beta}}-e^{x_{(j)}^{\beta}}\right)^{-1} \\
= & \frac{g_{2}\left(x_{(i)}\right)-g_{2}\left(x_{(j)}\right)}{g_{1}\left(x_{(i)}\right)-g_{1}\left(x_{(j)}\right)}=\frac{g_{2}^{\prime}\left(\xi_{2}\right)}{g_{1}^{\prime}\left(\xi_{1}\right)}>0 .
\end{aligned}
$$

Hence, we obtain $H^{\prime}(\beta)>0$, that is, $H(\beta)$ is increasing. Also $\lim _{\beta \rightarrow 0} H(\beta)=r(e-1)^{2} /[r(e-1)]^{2}=1 / r$, and $\lim _{\beta \rightarrow+\infty} H(\beta)=\lim _{\beta \rightarrow+\infty} \sum_{i=1}^{r}\left(1 /\left(\sum_{j=1}^{r}\left(\left(e^{x_{(j)}^{\beta}}-1\right) /\left(e^{x_{(i)}^{\beta}}\right.\right.\right.\right.$ $\left.-1)))^{2}\right)=1$ from

$$
\lim _{\beta \rightarrow+\infty} \sum_{j=1}^{r} \frac{e^{x_{(j)}^{\beta}}-1}{e^{x_{(i)}^{\beta}}-1}= \begin{cases}1 & i=r \\ +\infty, & i<r .\end{cases}
$$

Additionally, on the one hand, $\left(\sum_{i=1}^{r} b_{i, n}\right) /\left(\sum_{i=1}^{r} a_{i, n}\right)^{2} \geq$ $\left(\sum_{i=1}^{r} a_{i, n}^{2}+\sum_{i=1}^{r} d_{i, n}\right) / r \sum_{i=1}^{r} a_{i, n}^{2}>1 / r$, and on the other hand

$$
\begin{aligned}
& 2 \sum_{i>j} \sum_{i, n} a_{j, n} \\
&=2 \sum_{i>j} \sum_{j=1} \sum_{k=1}^{j} \sum_{l=1}^{j} \frac{1}{n-k+1} \times \frac{1}{n-l+1} \\
&=2 \sum_{i=1}^{r} \sum_{j=1}^{i-1} \sum_{k=1}^{i} \sum_{l=1}^{j} \frac{1}{n-k+1} \times \frac{1}{n-l+1} \\
&=2 \sum_{i=1}^{r} \sum_{k=1}^{i} \frac{1}{n-k+1} \sum_{j=1}^{i-1} \sum_{l=1}^{j} \frac{1}{n-l+1} \\
&=2 \sum_{i=1}^{r} \sum_{k=1}^{i} \frac{1}{n-k+1} \sum_{l=1}^{i-1} \sum_{j=l}^{i-1} \frac{1}{n-l+1} \\
&=2 \sum_{i=1}^{r} \sum_{k=1}^{i} \frac{1}{n-k+1} \sum_{l=1}^{i-1} \frac{i-l}{n-l+1} \\
& \geq \sum_{i=1}^{r}\left[\sum_{i=1}^{r} \sum_{k=1}^{i-1} \frac{i-k}{(n-k+1)^{2}}+\frac{1}{(n-k+1)^{2}}=\sum_{i=1}^{i} d_{i, n} .\right. \\
&(n-1)(n-i+2)
\end{aligned}
$$

So $1 / r<\left(\sum_{i=1}^{r} b_{i, n}\right) /\left(\sum_{i=1}^{r} a_{i, n}\right)^{2}=\left(\sum_{i=1}^{r} a_{i, n}^{2}+\sum_{i=1}^{r} d_{i, n}\right) /$ $\left(\sum_{i=1}^{r} a_{i, n}^{2}+2 \sum \sum_{i>j} a_{i, n} a_{j, n}\right) \leq 1$. Thus, the equation $H(\beta)=$ $\left(\sum_{i=1}^{r} b_{i, n}\right) /\left(\sum_{i=1}^{r} a_{i, n}\right)^{2}$ has a unique root in $\beta>0$.

\section{Conflict of Interests}

The authors declare that there is no conflict of interests regarding the publication of this paper. 


\section{Acknowledgments}

Wang and Xu's work was supported by the Grants from Innovation Program of Shanghai Municipal Education Commission (14YZ080, 14ZZ155). Wang's work was also supported by Shanghai Normal University SK201306. Sha's work was partially supported by NSF CMMI-0654417, and NIH NIMHD-8G12MD007592.

\section{References}

[1] U. Hjorth, "A reliability distribution with increasing, decreasing, constant and bathtub-shaped failure rates," Technometrics, vol. 22, no. 1, pp. 99-107, 1980.

[2] S. Rajarshi and M. B. Rajarshi, "Bathtub distributions: a review," Communications in Statistics: Theory and Methods, vol. 17, no. 8, pp. 2597-2621, 1988.

[3] G. S. Mudholkar and D. K. Srivastava, "Exponentiated Weibull family for analyzing bathtub failure-rate data," IEEE Transactions on Reliability, vol. 42, no. 2, pp. 299-302, 1993.

[4] J. Mi, "Bathtub failure rate and upside-down bathtub mean residual life," IEEE Transactions on Reliability, vol. 44, no. 3, pp. 388-391, 1995.

[5] M. Xie and C. D. Lai, "Reliability analysis using an additive Weibull model with bathtub-shaped failure rate function," Reliability Engineering and System Safety, vol. 52, no. 1, pp. 8793, 1996.

[6] Z. Chen, "A new two-parameter lifetime distribution with bathtub shape or increasing failure rate function," Statistics \& Probability Letters, vol. 49, no. 2, pp. 155-161, 2000.

[7] J. H. Cha, "An extended model for optimal burn-in procedures," IEEE Transactions on Reliability, vol. 55, no. 2, pp. 189-198, 2006.

[8] C. Xiong, "Inferences on a simple step-stress model with typeII censored exponential data," IEEE Transactions on Reliability, vol. 47, no. 2, pp. 142-146, 1998.

[9] N. Balakrishnan, E. Beutner, and M. Kateri, "Order restricted inference for exponential step-stress models," IEEE Transactions on Reliability, vol. 58, no. 1, pp. 132-142, 2009.

[10] W. Q. Meeker Jr., "A comparison of accelerated life test plans for Weibull and lognormal distributions and type-I censoring," Technometrics, vol. 26, no. 2, pp. 157-171, 1984.

[11] J.-W. Wu, C.-C. Wu, and M.-H. Tsai, "Optimal parameter estimation of the two-parameter bathtub-shaped lifetime distribution based on a type II right censored sample," Applied Mathematics and Computation, vol. 167, no. 2, pp. 807-819, 2005.

[12] Y. Tang, M. Xie, and T. N. Goh, "Statistical analysis of a Weibull extension model," Communications in Statistics: Theory and Methods, vol. 32, no. 5, pp. 913-928, 2003.

[13] B. S. Dhillon, "Life distributions," IEEE Transactions on Reliability, vol. 30, no. 5, pp. 457-460, 1981.

[14] J. Cao and K. Cheng, Introduction to Reliability Mathematics, Higher Education Press, Beijing, China, 2006.

[15] S. Malmquist, "On a property of order statistics from a rectangular distribution," Skandinavisk Aktuarietidskrift, vol. 33, pp. 214-222, 1950.

[16] B. Wang, "Statistical inference of Weibull distribution," Chinese Journal of Applied Probability and Statistics, vol. 8, no. 4, pp. 357364, 1992.

[17] N. R. Mann, R. E. Schafer, and N. D. Singpurwalla, Methods for Statistical Analysis of Reliability and Lifetime Data, John Wiley \& Sons, New York, NY, USA, 1974.
[18] M. Xie, Y. Tang, and T. N. Goh, "A modified Weibull extension with bathtub-shaped failure rate function," Reliability Engineering and System Safety, vol. 76, no. 3, pp. 279-285, 2002.

[19] L. Wang, Z. Dai, F. Yang, and X. Zheng, Research and Comments of Mathematical Analysis Problems, Science Press, Beijing, China, 1995. 


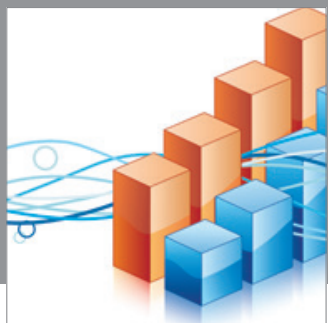

Advances in

Operations Research

mansans

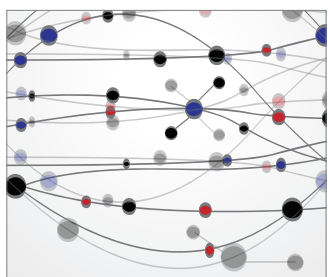

The Scientific World Journal
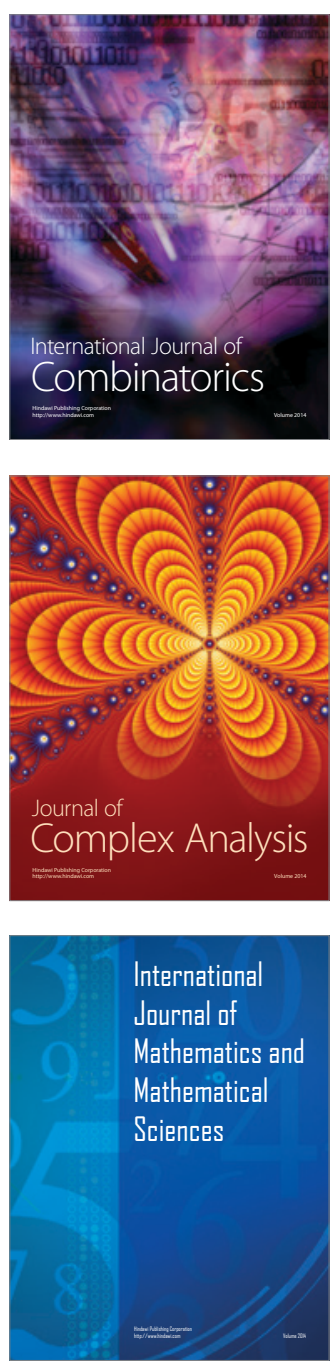
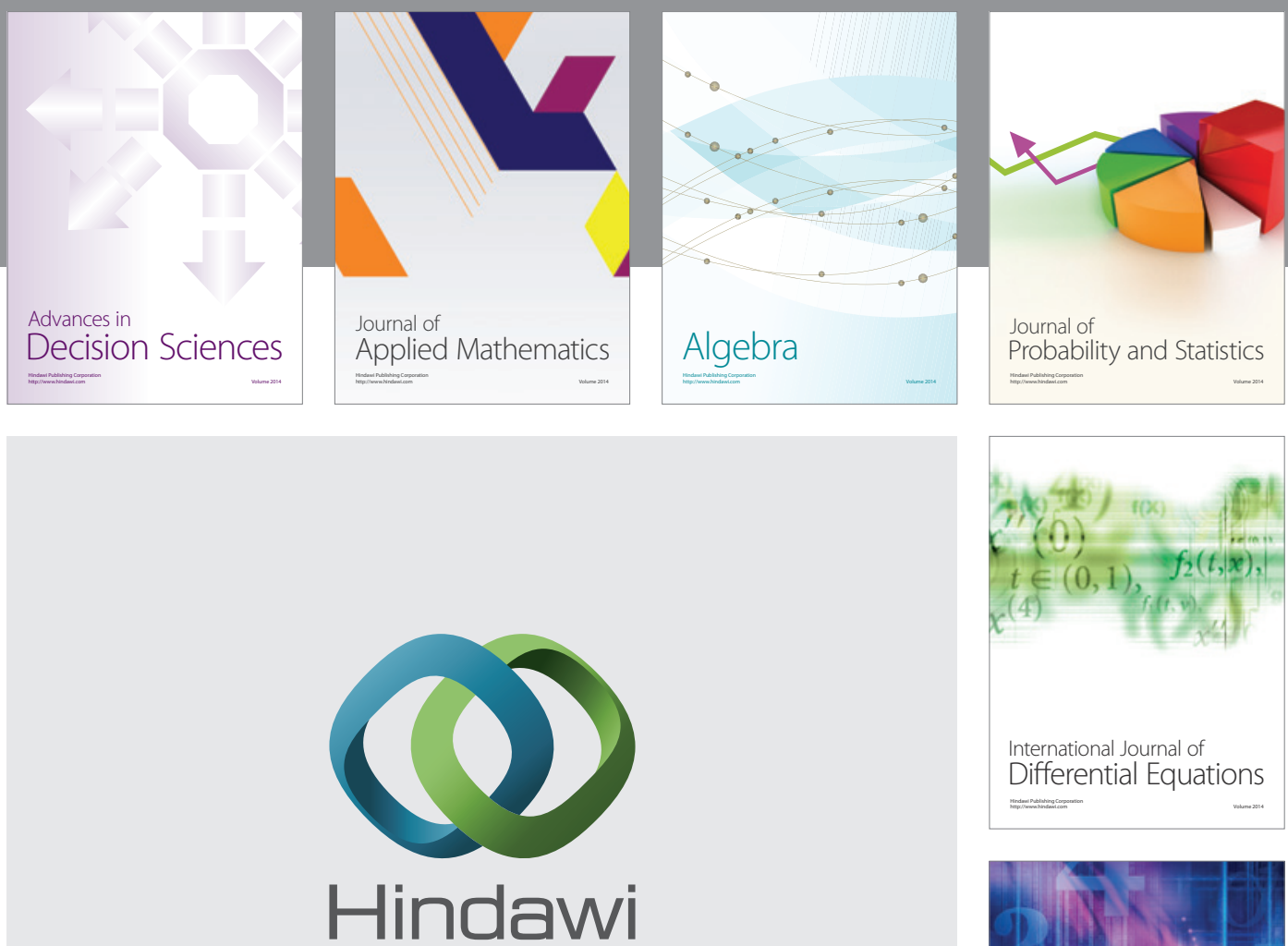

Submit your manuscripts at http://www.hindawi.com
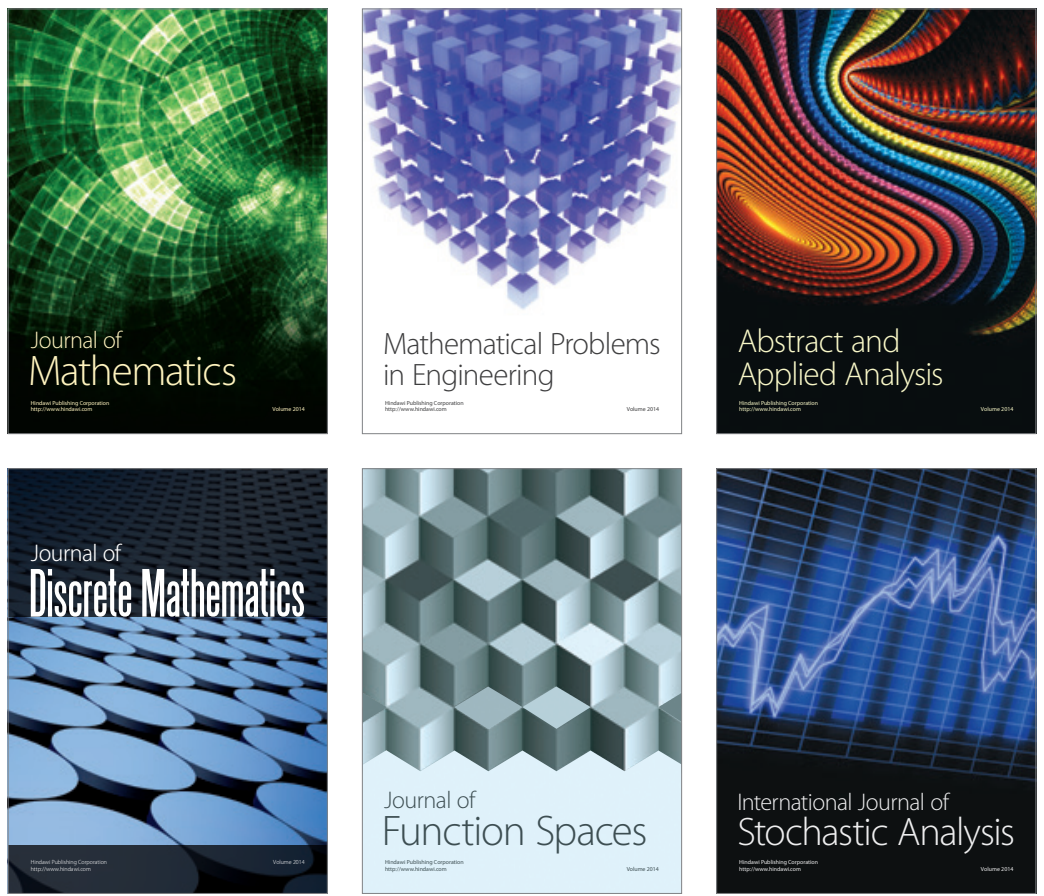

Journal of

Function Spaces

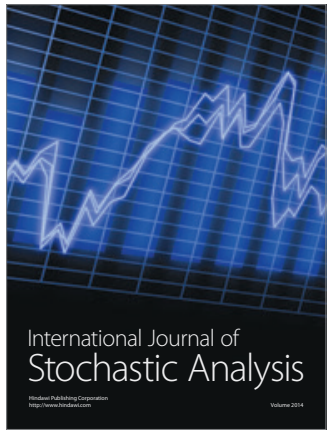

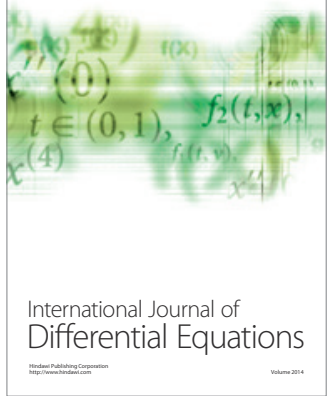
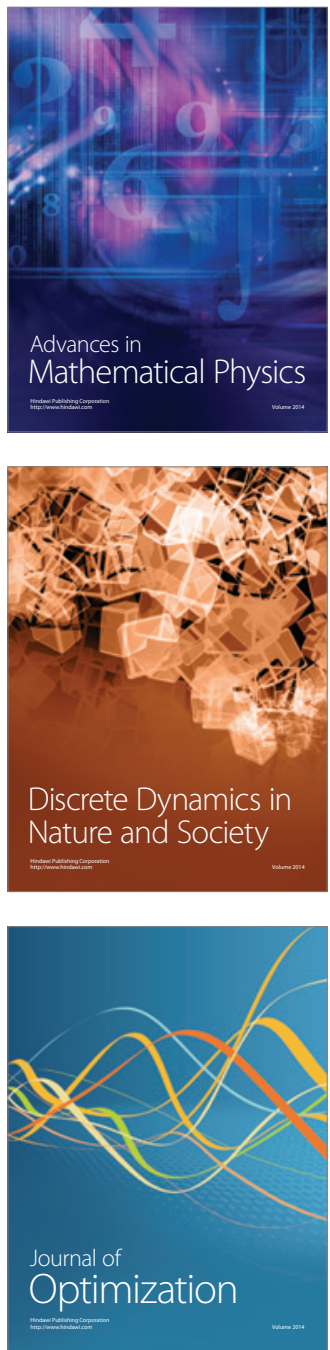